\title{
Decomposition systems for function spaces
}

\author{
by \\ G. KYRIAZIS (Nicosia)
}

\begin{abstract}
Let $\Theta:=\left\{\theta_{I}^{e}: e \in E, I \in D\right\}$ be a decomposition system for $L_{2}\left(\mathbb{R}^{d}\right)$ indexed over $D$, the set of dyadic cubes in $\mathbb{R}^{d}$, and a finite set $E$, and let $\widetilde{\Theta}:=\left\{\widetilde{\theta}_{I}^{e}\right.$ : $e \in E, I \in D\}$ be the corresponding dual functionals. That is, for every $f \in L_{2}\left(\mathbb{R}^{d}\right)$, $f=\sum_{e \in E} \sum_{I \in D}\left\langle f, \widetilde{\theta}_{I}^{e}\right\rangle \theta_{I}^{e}$. We study sufficient conditions on $\Theta, \widetilde{\Theta}$ so that they constitute a decomposition system for Triebel-Lizorkin and Besov spaces. Moreover, these conditions allow us to characterize the membership of a distribution $f$ in these spaces by the size of the coefficients $\left\langle f, \widetilde{\theta}_{I}^{e}\right\rangle, e \in E, I \in D$. Typical examples of such decomposition systems are various wavelet-type unconditional bases for $L_{2}\left(\mathbb{R}^{d}\right)$, and more general systems such as affine frames.
\end{abstract}

1. Introduction. Let $E$ be a finite set and $D$ be the family of dyadic cubes in $\mathbb{R}^{d}$. Given a decomposition system $\Theta:=\left\{\theta_{I}^{e}: e \in E, I \in D\right\}$, for $L_{2}\left(\mathbb{R}^{d}\right)$ with dual functionals $\widetilde{\Theta}:=\left\{\widetilde{\theta}_{I}^{e}: e \in E, I \in D\right\}$ our goal is to study sufficient conditions on $\Theta, \widetilde{\Theta}$ so that they constitute a decomposition system for the homogeneous Triebel-Lizorkin and Besov spaces. That is, every distribution $f$ in the above spaces can be expressed in the form

$$
f=\sum_{e \in E} \sum_{I \in D}\left\langle f, \widetilde{\theta}_{I}^{e}\right\rangle \theta_{I}^{e} .
$$

Moreover, we are interested in characterizing the membership of a distribution $f$ in the Triebel-Lizorkin and Besov spaces by the size of the coefficients $\left\langle f, \widetilde{\theta}_{I}^{e}\right\rangle, e \in E, I \in D$. Typical examples of such systems are various unconditional bases for $L_{2}\left(\mathbb{R}^{d}\right)$ such as the biorthogonal wavelet bases, the bases constructed in [Pet] or even the affine frames of $L_{2}\left(\mathbb{R}^{d}\right)$.

To describe our results we first introduce the standard multi-index notation. For $x=\left(x_{1}, \ldots, x_{d}\right) \in \mathbb{R}^{d}$ and $\alpha=\left(\alpha_{1}, \ldots, \alpha_{d}\right) \in \mathbb{N}^{d}(\mathbb{N}:=$ $\{n: n \geq 0\}, d \geq 1)$, we let $|x|:=\sqrt{x_{1}^{2}+\ldots+x_{d}^{2}}, x^{\alpha}:=x_{1}^{\alpha_{1}} \ldots x_{d}^{\alpha_{d}}$, $|\alpha|:=\alpha_{1}+\ldots+\alpha_{d}, \alpha !=\alpha_{1} ! \ldots \alpha_{d} !$, and $(\cdot)^{(\alpha)}:=\partial^{|\alpha|}(\cdot) / \partial x_{1}^{\alpha_{1}} \ldots \partial x_{d}^{\alpha_{d}}$.

2000 Mathematics Subject Classification: 41A17, 41A20, 42B25, 42C15.

Key words and phrases: unconditional bases, wavelets, frames, Besov spaces, TriebelLizorkin spaces. 
Also for every $x \in \mathbb{R}$ we use $[x]$ for the greatest integer which is less than or equal to $x$.

We denote by $\mathcal{S}:=\mathcal{S}\left(\mathbb{R}^{d}\right)$ the Schwartz space of infinitely differentiable, rapidly decreasing functions on $\mathbb{R}^{d}$ and by $\mathcal{S}^{\prime}:=\mathcal{S}^{\prime}\left(\mathbb{R}^{d}\right)$ its dual, the space of tempered distributions. For every $k \in \mathbb{N}$ we define

$$
\mathcal{S}_{k}:=\mathcal{S}_{k}\left(\mathbb{R}^{d}\right):=\left\{\eta \in \mathcal{S}: \int \eta(x) x^{\alpha} d x=0,|\alpha| \leq k\right\}
$$

and we identify the dual space of $\mathcal{S}_{k}$ with $\mathcal{S}^{\prime} / \mathcal{P}_{k}$, the space of equivalence classes of distributions modulo polynomials of degree $\leq k$.

Similarly, we use the notation

$$
\mathcal{S}_{\infty}:=\mathcal{S}_{\infty}\left(\mathbb{R}^{d}\right):=\left\{\eta \in \mathcal{S}: \int \eta(x) x^{\alpha} d x=0, \forall \alpha \in \mathbb{N}^{d}\right\},
$$

and we denote by $\mathcal{S}^{\prime} / \mathcal{P}$ the space of equivalence classes of distributions modulo polynomials.

For any $m \in \mathbb{Z}, k \in \mathbb{Z}^{d}$, the dyadic cube $I_{m, k} \in D$ is defined by $I_{m, k}:=$ $2^{-m}\left(k+[0,1)^{d}\right)$. If $I=I_{m, k}$ we use $x_{I}$ for its "lower-left" vertex $2^{-m} k$, and $|I|$ for its volume. Also by $D_{m}, m \in \mathbb{Z}$, we denote the collection of all cubes $I \in D$ of sidelength $\ell(I)=2^{-m}$. We use $\langle f, \eta\rangle$ for the standard inner product $\int f \bar{\eta}$ of two functions, when this makes sense, and the same notation is employed for the action of a distribution $f \in \mathcal{S}^{\prime}$ on $\bar{\eta} \in \mathcal{S}$.

Also we denote the Fourier transform of an integrable function $f$ by

$$
\widehat{f}(\xi):=\int_{\mathbb{R}^{d}} f(x) e^{-i x \cdot \xi} d x .
$$

By duality the Fourier transform is extended to $\mathcal{S}^{\prime}$.

Let now $B:=\left\{\theta_{I}^{e}, \widetilde{\theta}_{I}^{e}: e \in E, I \in D\right\}$, be a family of functions on $\mathbb{R}^{d}$ which satisfies the following assumptions:

A. $B$ forms a decomposition system for $L_{2}\left(\mathbb{R}^{d}\right)$, i.e., for every $f \in$ $L_{2}\left(\mathbb{R}^{d}\right)$,

$$
f=\sum_{e \in E} \sum_{I \in D}\left\langle f, \widetilde{\theta}_{I}^{e}\right\rangle \theta_{I}^{e}
$$

in the sense of $L_{2}\left(\mathbb{R}^{d}\right)$.

B1. For every $e \in E, I \in D$,

$$
\begin{aligned}
& \left|\left(\theta_{I}^{e}\right)^{(\alpha)}(x)\right| \leq C|I|^{-1 / 2-|\alpha| / d}\left(1+\frac{\left|x-x_{I}\right|}{\ell(I)}\right)^{-M_{\Theta}}, \quad|\alpha| \leq r_{\Theta}, \\
& \left|\left(\widetilde{\theta}_{I}^{e}\right)^{(\alpha)}(x)\right| \leq C|I|^{-1 / 2-|\alpha| / d}\left(1+\frac{\left|x-x_{I}\right|}{\ell(I)}\right)^{-M_{\widetilde{\Theta}},} \quad|\alpha| \leq r_{\widetilde{\Theta}},
\end{aligned}
$$

where $M_{\Theta}, M_{\widetilde{\Theta}}>0$. 
B2. For every $e \in E, I \in D$,

$$
\begin{array}{ll}
\int_{\mathbb{R}^{d}} x^{\alpha} \theta_{I}^{e}(x) d x=0, & |\alpha| \leq r_{\widetilde{\Theta}}-1, \\
\int_{\mathbb{R}^{d}} x^{\alpha} \widetilde{\theta}_{I}^{e}(x) d x=0, & |\alpha| \leq r_{\Theta}-1 .
\end{array}
$$

By varying the smoothness and decay parameters $r_{\Theta}, r_{\widetilde{\Theta}}$ and $M_{\Theta}, M_{\widetilde{\Theta}}$, one can prove that $B$ forms a decomposition system for a host of distribution spaces such as $L_{p}:=L_{p}\left(\mathbb{R}^{d}\right)(1<p<\infty), H_{p}:=H_{p}\left(\mathbb{R}^{d}\right)(0<p \leq 1)$, the potential spaces $H_{p}^{s}, s>0,1<p<\infty$, or the more general homogeneous Triebel-Lizorkin and Besov spaces $\dot{F}_{p q}^{s}$ and $\dot{B}_{p q}^{s}$. Moreover, the size of a distribution $f$ measured in the (quasi)norms of these spaces can be determined by (quasi)norms applied to the sequence of coefficients $\left\langle f, \widetilde{\theta}_{I}^{e}\right\rangle$, $e \in E, I \in D$. We recall (see $[\mathrm{T}]$ ) that for $1<p<\infty, \dot{F}_{p 2}^{0} \approx L_{p}$, while for $0<p \leq 1, \dot{F}_{p 2}^{0} \approx H_{p}$. Also, for $s>0,1<p<\infty, \dot{F}_{p 2}^{s} \approx H_{p}^{s}$, and for integer values of $s, \dot{F}_{p 2}^{s} \approx W_{p}^{s}$, the Sobolev space equipped with its seminorm (here $\approx$ means that the spaces have equivalent (quasi)norms - see below).

In particular, we shall prove (Theorem 4.1) that if $r_{\Theta}, r_{\widetilde{\Theta}}$ and $M_{\Theta}, M_{\widetilde{\Theta}}$ are sufficiently large (depending on the parameters $s \in \mathbb{R}, 0<p<\infty$, and $0<q \leq \infty)$, then for every $f \in \dot{F}_{p q}^{s}$,

$$
f=\sum_{e \in E} \sum_{I \in D} \widetilde{a}_{I}^{e}(f) \theta_{I}^{e}, \quad \widetilde{a}_{I}^{e}(f):=\left\langle f, \widetilde{\theta}_{I}^{e}\right\rangle,
$$

where the convergence is considered in the distributional sense (and in the sense of $\dot{F}_{p q}^{s}$ when $q \neq \infty$ ). Moreover, the following characterization holds:

$$
\|f\|_{\dot{F}_{p q}^{s}} \approx \sum_{e \in E}\left\|\left(\sum_{I \in D}\left(|I|^{-s / d}\left|\widetilde{a}_{I}^{e}(f)\right| \widetilde{\chi}_{I}\right)^{q}\right)^{1 / q}\right\|_{L_{p}},
$$

where $\tilde{\chi}_{I}:=|I|^{-1 / 2} \chi_{I}$ is the characteristic function of $I$ normalized in $L_{2}$. Here we have adopted the notation $A \approx B$ which means that there exist constants $C_{1}, C_{2}>0$ such that $C_{1} A \leq B \leq C_{2} A$. The equivalence constants $C_{1}$ and $C_{2}$ in (1.4) depend on $d, p, q$, and $s$. On other occasions, the reader will have to consult the text to understand the parameters on which the equivalence constants depend on. Throughout the paper, the constants are denoted by $C$ and they may vary at every occurrence.

Similarly (for suitable $r_{\Theta}, r_{\widetilde{\Theta}}$ and $M_{\Theta}, M_{\widetilde{\Theta}}$ ) we shall prove (Theorem 4.2) that for every $f \in \dot{B}_{p q}^{s}, s \in \mathbb{R}, 0<p, q \leq \infty$, the representation (1.3) holds in the distributional sense (and in the sense of $\dot{B}_{p q}^{s}$ when $p, q \neq \infty$ ). Also 


$$
\|f\|_{\dot{B}_{p q}^{s}} \approx \sum_{e \in E}\left(\sum_{m \in \mathbb{Z}}\left(\sum_{I \in D_{m}}\left(|I|^{-s / d+1 / p-1 / 2}\left|\widetilde{a}_{I}^{e}(f)\right|\right)^{p}\right)^{q / p}\right)^{1 / q}
$$

with the usual modifications when $q=\infty$ or $p=\infty$.

However, in the literature people are often interested in obtaining unconditional bases, rather than simple decomposition systems for various function spaces. In particular, biorthogonal wavelet bases have gained great popularity over the last decade. This is primarily due to their applications in many fields such as statistics [Do], image processing [DJKP], Fourier and functional analysis. One of the main features of the wavelet bases, which to some degree explains their popularity, is that, similarly to (1.4) and (1.5), they frequently lead to simple characterizations of various spaces whose membership is expressed in terms of (quasi)norms applied to the sequence of coefficients with respect to the basis. Thus, we would like to develop a parallel theory for extending an unconditional basis of $L_{2}\left(\mathbb{R}^{d}\right)$ to an unconditional basis for the Triebel-Lizorkin and Besov spaces as well.

This comes at no significant cost in our setting. We only need to replace assumption $\mathbf{A}$ above with the following one:

$\mathbf{A}^{\prime}$. (i) With $\delta$ denoting the Kronecker delta,

$$
\left\langle\widetilde{\theta}_{I}^{e}, \theta_{J}^{e^{\prime}}\right\rangle=\delta_{I, J} \delta_{e, e^{\prime}}, \quad I, J \in D, e, e^{\prime} \in E .
$$

(ii) For every $f \in L_{2}\left(\mathbb{R}^{d}\right)$,

$$
f=\sum_{e \in E} \sum_{I \in D}\left\langle f, \widetilde{\theta}_{I}^{e}\right\rangle \theta_{I}^{e}, \quad\|f\|_{L_{2}} \approx\left(\sum_{e \in E} \sum_{I \in D}\left|\left\langle f, \widetilde{\theta}_{I}^{e}\right\rangle\right|^{2}\right)^{1 / 2} .
$$

Technically speaking, these systems will be bases for the Triebel-Lizorkin and Besov spaces provided that $p, q \neq \infty$, since the convergence of the corresponding series then takes place in the (quasi)norm of the space in question. However, with a slight abuse of the terminology we use the word bases even if $p$ or $q$ is $\infty$, where the convergence is considered only in distributional sense. We recall that the characterizations of $L_{p}, H_{p}$ and the potential spaces in terms of orthogonal wavelet bases have been established by Meyer $[\mathrm{M}]$; we also refer the reader to $[\mathrm{HW}]$ for a complete account of these cases. On the other hand Besov spaces with respect to orthogonal wavelets have been studied in $[\mathrm{M}]$, and in $[\mathrm{B}],[\mathrm{K}]$ for various subcases of the indices and under different assumptions. Characterizations, though, for the general TriebelLizorkin spaces can been found in [FJW] only in the special case of Meyer's orthogonal wavelets which belong in $\mathcal{S}$ and their Fourier transform vanishes in a neighborhood of the origin.

In contrast, as a consequence of our results we establish the wavelet characterizations of homogeneous Triebel-Lizorkin (Proposition 4.1) and Besov spaces (Proposition 4.2) in terms of general biorthogonal wavelets for the full 
range of the indices $s, p, q$. One of the advantages of this approach is that it allows us to disassociate the conditions imposed on the wavelet set $\Psi$ from the ones imposed on their dual set $\widetilde{\Psi}$ (see definitions below) and thus the various regularity and decay conditions of one family do not affect the other.

We also mention that in certain applications, such as image processing, people are interested in unconditional bases for $L_{2}\left(\mathbb{R}^{d}\right)$ that are indexed not by the set $D$ of all dyadic cubes, but by $D_{+}:=\bigcup_{m \geq 0} D_{m}$, the set of dyadic cubes with sidelength $\leq 1$.

It turns out that these bases are tailor-made for the characterization of the inhomogeneous Triebel-Lizorkin and Besov spaces $F_{p q}^{s}, B_{p q}^{s}$. A brief but comprehensive analysis of these results is presented in $\S 5$ solely within the framework of biorthogonal wavelet bases, for notational reasons.

In dealing with homogeneous spaces, an important point that one needs to be careful about is the meaning of the decomposition formula (1.3). This is a rather delicate issue, and it turns out that the convergence as well as the equality in (1.3) take place in the topology of $\mathcal{S}^{\prime} / \mathcal{P}_{k}$, where $k \in \mathbb{N}$ depends on the space under consideration. To address this one needs to take a closer look at Calderón's reproducing formula. A complete account of these results is presented in $\S 2$ and in Appendix I.

Recapitulating, our primary goal in this paper is to present a general method for extending decomposition systems of $L_{2}\left(\mathbb{R}^{d}\right)$, which due to the geometric structure of this space are easier to construct, to decomposition systems for both scales of Triebel-Lizorkin and Besov spaces. For this, our main tool will be the $\phi$-transform (see (2.16) below) and the powerful machinery developed in [FJ2].

1.1. Biorthogonal wavelets. Biorthogonal wavelet bases were introduced by Cohen, Daubechies and Feauveau [CDF]. Their construction begins with two univariate scaling functions $\varphi$ and $\widetilde{\varphi}$ whose shifts are in duality:

$$
\left\langle\varphi(\cdot-k), \widetilde{\varphi}\left(\cdot-k^{\prime}\right)\right\rangle=\delta_{k, k^{\prime}}, \quad k, k^{\prime} \in \mathbb{Z} .
$$

Associated to each of the scaling functions are mother wavelets $\psi$ and $\widetilde{\psi}$. These functions can be used to generate a basis for $L_{2}\left(\mathbb{R}^{d}\right)$ as follows. We define $\psi^{0}:=\varphi, \widetilde{\psi}^{0}:=\widetilde{\varphi}, \psi^{1}:=\psi$ and $\widetilde{\psi}^{1}:=\widetilde{\psi}$. Let $E_{0}$ denote the collection of all vertices of the unit cube $[0,1]^{d}$ and $E:=E_{0} \backslash\{\mathbf{0}\}(\mathbf{0}=(0, \ldots, 0))$. For each vertex $e=\left(e_{1}, \ldots, e_{d}\right) \in E_{0}$, we define the multivariate functions

$$
\begin{aligned}
& \psi^{e}\left(x_{1}, \ldots, x_{d}\right):=\psi^{e_{1}}\left(x_{1}\right) \ldots \psi^{e_{d}}\left(x_{d}\right), \\
& \widetilde{\psi}^{e}\left(x_{1}, \ldots, x_{d}\right):=\widetilde{\psi}^{e_{1}}\left(x_{1}\right) \ldots \widetilde{\psi}^{e_{d}}\left(x_{d}\right) .
\end{aligned}
$$

Following the wavelet literature, for every $I \in D$ we also define

$$
\psi_{I}^{e}(\cdot):=|I|^{-1 / 2} \psi^{e}\left(\frac{\cdot-x_{I}}{\ell(I)}\right), \quad \widetilde{\psi}_{I}^{e}(\cdot):=|I|^{-1 / 2} \psi^{e}\left(\frac{\cdot-x_{I}}{\ell(I)}\right) .
$$


(A word of caution: this notation will be used only for specific functions such as the wavelets and in the $\phi$-transform (see (2.16) below) and not necessarily for general functions as the ones in the sets $\Theta$ and $\widetilde{\Theta}$ considered earlier.)

Then the collection of functions

$$
W:=\left\{\psi_{I}^{e}, \widetilde{\psi}_{I}^{e}: I \in D, e \in E\right\}
$$

constitutes a Riesz basis for $L_{2}\left(\mathbb{R}^{d}\right)$ with

$$
\left\langle\widetilde{\psi}_{I}^{e}, \psi_{J}^{e^{\prime}}\right\rangle=\delta_{I, J} \delta_{e, e^{\prime}}
$$

In particular, for every $f \in L_{2}\left(\mathbb{R}^{d}\right)$,

$$
f=\sum_{e \in E} \sum_{I \in D} \widetilde{c}_{I}^{e}(f) \psi_{I}^{e}=\sum_{e \in E} \sum_{I \in D} c_{I}^{e}(f) \widetilde{\psi}_{I}^{e}
$$

where

$$
\widetilde{c}_{I}^{e}(f):=\left\langle f, \widetilde{\psi}_{I}^{e}\right\rangle, \quad c_{I}^{e}(f):=\left\langle f, \psi_{I}^{e}\right\rangle,
$$

and in addition

$$
\|f\|_{L_{2}\left(\mathbb{R}^{d}\right)} \approx\left(\sum_{e \in E} \sum_{I \in D}\left|\widetilde{c}_{I}^{e}(f)\right|^{2}\right)^{1 / 2} \approx\left(\sum_{e \in E} \sum_{I \in D}\left|c_{I}^{e}(f)\right|^{2}\right)^{1 / 2} .
$$

If $\varphi=\widetilde{\varphi}$ then $\left\{\psi_{I}^{e}: I \in D, e \in E\right\}$ constitutes an orthonormal basis for $L_{2}\left(\mathbb{R}^{d}\right)$.

Alternatively, one can construct biorthogonal bases indexed by the set $E_{0}$ and the dyadic cubes in $D_{+}$, namely

$$
W_{0}:=\left\{\psi_{I}^{\mathbf{0}}, \widetilde{\psi}_{I}^{\mathbf{0}}: I \in D_{0}\right\} \cup\left\{\psi_{I}^{e}, \widetilde{\psi}_{I}^{e}: I \in D_{+}, e \in E\right\} .
$$

In this case for instance, for every $f \in L_{2}\left(\mathbb{R}^{d}\right)$ we have

$$
f=\sum_{I \in D_{0}} \widetilde{c}_{I}^{\mathbf{0}}(f) \psi_{I}^{\mathbf{0}}+\sum_{e \in E} \sum_{I \in D_{+}} \widetilde{c}_{I}^{e}(f) \psi_{I}^{e},
$$

where $\widetilde{c}_{I}^{e}(f):=\left\langle f, \widetilde{\psi}_{I}^{e}\right\rangle, e \in E_{0}$, and

$$
\|f\|_{L_{2}\left(\mathbb{R}^{d}\right)} \approx\left(\sum_{I \in D_{0}}\left|\widetilde{c}_{I}^{0}(f)\right|^{2}\right)^{1 / 2}+\left(\sum_{e \in E} \sum_{I \in D_{+}}\left|\widetilde{c}_{I}^{e}(f)\right|^{2}\right)^{1 / 2} .
$$

Standard assumptions on the sets

$$
\Psi:=\left\{\psi^{e}: e \in E_{0}\right\}, \quad \widetilde{\Psi}:=\left\{\widetilde{\psi}^{e}: e \in E_{0}\right\}
$$

include:

B1'. $\Psi \subset C^{r_{\Psi}}\left(\mathbb{R}^{d}\right), \widetilde{\Psi} \subset C^{r_{\widetilde{\Psi}}}\left(\mathbb{R}^{d}\right), r_{\Psi}, r_{\widetilde{\Psi}} \in \mathbb{N}_{+}$, and for $e \in E_{0}$,

$$
\begin{array}{ll}
\left|\left(\psi^{e}\right)^{(\alpha)}(x)\right| \leq C(1+|x|)^{-M_{\Psi}}, & |\alpha| \leq r_{\Psi}, \\
\left|\left(\widetilde{\psi}^{e}\right)^{(\alpha)}(x)\right| \leq C(1+|x|)^{-M_{\widetilde{\Psi}},} & |\alpha| \leq r_{\widetilde{\Psi}},
\end{array}
$$

where $M_{\Psi}, M_{\widetilde{\Psi}}>0$. 
$\mathbf{B 2}^{\prime}$. For $e \in E$,

$$
\begin{array}{rlrl}
\int_{\mathbb{R}^{d}} x^{\alpha} \psi^{e}(x) d x=0, & |\alpha| \leq r_{\widetilde{\Psi}}-1, \\
\int_{\mathbb{R}^{d}} x^{\alpha} \widetilde{\psi}^{e}(x) d x=0, & & |\alpha| \leq r_{\Psi}-1 .
\end{array}
$$

For instance, starting with Meyer's univariate scaling function and wavelet (see $[\mathrm{M}]$ ) one obtains an orthonormal basis of functions from $\mathcal{S}_{\infty}$ which satisfy $\mathbf{B} 1^{\prime}-\mathbf{B} 2^{\prime}$ for any selection of the parameters $r_{\Psi}$ and $M_{\Psi}$. B1',

We note that $\psi^{\mathbf{0}}(x)=\varphi\left(x_{1}\right) \ldots \varphi\left(x_{d}\right), \widetilde{\psi}^{\mathbf{0}}(x)=\widetilde{\varphi}\left(x_{1}\right) \ldots \widetilde{\varphi}\left(x_{d}\right)$, and from

$$
\begin{array}{ll}
\left|\left(\psi^{\mathbf{0}}\right)^{(\alpha)}(x)\right| \leq C(1+|x|)^{-M_{\Psi}}, & |\alpha| \leq r_{\Psi}, \\
\left|\left(\widetilde{\psi}^{\mathbf{0}}\right)^{(\alpha)}(x)\right| \leq C(1+|x|)^{-M_{\widetilde{\Psi}},} & |\alpha| \leq r_{\widetilde{\Psi}},
\end{array}
$$

(no zero moments are required).

If $\Psi, \widetilde{\Psi}$ satisfies $\mathbf{B} 1^{\prime}-\mathbf{B 2}{ }^{\prime}$ then from (1.6) it becomes clear that the biorthogonal family $W$ satisfies assumptions $\mathbf{B} \mathbf{1}-\mathbf{B 2}$, while $\mathbf{A}^{\prime}$ is inherited by $W$ from its construction. Therefore, for appropriate smoothness and decay parameters $r_{\Psi}, r_{\widetilde{\Psi}}$ and $M_{\Psi}, M_{\widetilde{\Psi}}$, we see that $W$ forms an unconditional basis for the homogeneous Triebel-Lizorkin and Besov spaces $\dot{F}_{p q}^{s}$ and $\dot{B}_{p q}^{s}$.

1.2. Outline of the paper. In $\S 2$ we give the definitions of the homogeneous Triebel-Lizorkin and Besov spaces and we also give a complete account of the topology of $\mathcal{S}^{\prime} / \mathcal{P}_{k}, k \in \mathbb{N}$. In $\S 3$ we study the boundedness of matrix operators on the sequence spaces $\dot{f}_{p q}^{s}$ and $\dot{b}_{p q}^{s}$ (see definitions below). This type of problems have been studied, in the case of $\dot{f}$-spaces, by Frazier and Jawerth [FJ2] who used duality arguments; here instead we use a straightforward approach and our assumptions are tailor-made for our results. In $\S 4$ we prove the main results regarding the homogeneous spaces while the results on the inhomogeneous spaces are briefly presented in $\S 5$. Finally, in Appendix I we present some results related to Calderón's reproducing formula, while in Appendix II we have included some useful lemmas which will be used throughout the paper.

2. Triebel-Lizorkin and Besov spaces. Let $\phi \in \mathcal{S}$ be such that the family of functions $\phi_{\nu}(\cdot):=2^{\nu d} \phi\left(2^{\nu} \cdot\right), \nu \in \mathbb{Z}$, has the following properties:

$$
\begin{aligned}
& \text { (i) } \operatorname{supp} \widehat{\phi}_{\nu} \subset\left\{2^{\nu-1} \leq|\xi| \leq 2^{\nu+1}\right\}, \\
& \text { (ii) }\left|\widehat{\phi}_{\nu}^{(\beta)}(\xi)\right| \leq C 2^{-\nu|\beta|}, \quad \beta \in \mathbb{Z}^{d}, \\
& \text { (iii) } \sum_{\nu \in \mathbb{Z}}\left|\widehat{\phi}_{\nu}(\xi)\right|^{2}=1, \quad \xi \in \mathbb{R}^{d} \backslash\{0\} .
\end{aligned}
$$


For $s \in \mathbb{R}, 0<p<\infty, 0<q \leq \infty$, the homogeneous Triebel-Lizorkin space $\dot{F}_{p q}^{s}$ is defined to be the set of all $f \in \mathcal{S}^{\prime} / \mathcal{P}$ such that

$$
\|f\|_{\dot{F}_{p q}^{s}}:=\left\|\left(\sum_{\nu \in \mathbb{Z}}\left(2^{\nu s}\left|\phi_{\nu} * f\right|\right)^{q}\right)^{1 / q}\right\|_{L_{p}}<\infty
$$

(with the usual modification for $q=\infty$ ).

In a similar vein, for $s \in \mathbb{R}, 0<p, q \leq \infty$, the homogeneous Besov space $\dot{B}_{p q}^{s}$ is defined to be the set of all $f \in \mathcal{S}^{\prime} / \mathcal{P}$ such that

$$
\|f\|_{\dot{B}_{p q}^{s}}:=\left(\sum_{\nu \in \mathbb{Z}}\left(2^{\nu s}\left\|\phi_{\nu} * f\right\|_{L_{p}}\right)^{q}\right)^{1 / q}<\infty .
$$

Although the above definitions are independent of $\phi$ (different $\phi$ 's give rise to equivalent (quasi)norms), the fact that $\left\{\widehat{\phi}_{\nu}\right\}_{\nu \in \mathbb{N}}$ is a partition of unity, in the sense of (2.1)(iii), is of paramount importance in the definition of these spaces and will play a critical role in what follows. For instance in the case of $L_{2}\left(\mathbb{R}^{d}\right)$, applying the inverse Fourier transform we can easily deduce from (2.1)(iii) that

$$
\sum_{\nu \in \mathbb{Z}} \widetilde{\phi}_{\nu} * \phi_{\nu} * f=f
$$

$\left(\widetilde{\phi}_{\nu}(x):=\overline{\phi_{\nu}(-x)}\right)$ in the sense of $L_{2}\left(\mathbb{R}^{d}\right)$. (2.4) is the so-called Calderón's reproducing formula. Trying to explore its meaning in the various function spaces that we shall consider it is natural to ask whether the series $\sum_{\nu \in \mathbb{Z}} \widetilde{\phi}_{\nu} *$ $\phi_{\nu} * f$ is meaningful at least in the distributional sense. It turns out that if $f \in \mathcal{S}^{\prime}$ then the sum $\sum_{\nu=0}^{\infty} \widetilde{\phi}_{\nu} * \phi_{\nu} * f$ provides no difficulty since it converges in the sense of $\mathcal{S}^{\prime}$. To see this, using the continuity of the Fourier transform on $\mathcal{S}^{\prime}$ and applying the regularity theorem to $\widehat{f} \in \mathcal{S}^{\prime}$, we find that there exists $r \in \mathbb{N}$ such that for every $\eta \in \mathcal{S}$,

$$
|\langle\widehat{f}, \widehat{\eta}\rangle| \leq C \sum_{|\alpha| \leq r} \int_{\mathbb{R}^{d}}|\xi|^{r}\left|\widehat{\eta}^{(\alpha)}(\xi)\right| d \xi .
$$

From this estimate it follows easily that $\sum_{\nu \geq 0}\left|\left\langle\left(\widetilde{\phi}_{\nu} * \phi_{\nu} * f\right)^{\wedge}, \widehat{\eta}\right\rangle\right|<\infty$ (see [Pee, p. 52]).

However, the lower part of the series in (2.4), namely $\sum_{\nu<0} \widetilde{\phi}_{\nu} * \phi_{\nu} * f$, in general, does not converge in $\mathcal{S}^{\prime}$ and then one is forced to consider its convergence in the space $\mathcal{S}^{\prime} / \mathcal{P}$. The following instructive example illustrates this fact:

ExAmple. We let $f_{s}, s>0$, be the univariate distribution in $(\mathcal{S}(\mathbb{R}))^{\prime}$ defined by 


$$
\left\langle\widehat{f_{s}}, \widehat{\eta}\right\rangle:=\int_{0}^{s} \frac{\widehat{\eta}(\xi)-\widehat{\eta}(0)}{\xi} d \xi+\int_{s}^{\infty} \frac{\widehat{\eta}(\xi)}{\xi} d \xi, \quad \eta \in \mathcal{S}(\mathbb{R}) .
$$

Let also $\widehat{\theta} \in \mathcal{S}(\mathbb{R})$ be such that $\widehat{\theta}=1$ for $|\xi| \leq 2$, and $\widehat{\theta}=0$ for $|\xi| \geq 4$. From Parseval's formula we get

$$
\sum_{\nu \leq 0}\left\langle\widetilde{\phi}_{\nu} * \phi_{\nu} * f_{s}, \theta\right\rangle=\sum_{\nu \leq 0} \int_{0}^{\infty} \frac{\left|\widehat{\phi}_{\nu}(\xi)\right|^{2} \widehat{\theta}(\xi)}{\xi} d \xi=\sum_{\nu \leq 0} \int_{0}^{\infty} \frac{\left|\widehat{\phi}\left(\xi / 2^{\nu}\right)\right|^{2}}{\xi} d \xi=\infty
$$

where $\left\{\phi_{\nu}\right\}_{\nu}$ is the univariate version of (2.1). This demonstrates that the series $\sum_{\nu \leq 0} \widetilde{\phi}_{\nu} * \phi_{\nu} * f_{s}$ does not converge in $(\mathcal{S}(\mathbb{R}))^{\prime}$. A moment's reflection reveals however that if we consider the action of $\sum_{\nu \leq 0} \widetilde{\phi}_{\nu} * \phi_{\nu} * f_{s}$ on the subspace

$$
\mathcal{S}_{0}(\mathbb{R}):=\left\{\eta \in \mathcal{S}(\mathbb{R}): \int \eta(x) d x=0\right\},
$$

then we can get around the singularity at the origin by using the estimate $|\widehat{\eta}(\xi)| \leq C|\xi|(\widehat{\eta}(0)=0)$. In other words, if we consider $f_{s}$ as an element of the topological dual of $\mathcal{S}_{0}(\mathbb{R})$ instead of $\mathcal{S}(\mathbb{R})$, then $\sum_{\nu \in \mathbb{Z}} \widetilde{\phi}_{\nu} * \phi_{\nu} * f_{s}$ defines a distribution in $\left(\mathcal{S}_{0}(\mathbb{R})\right)^{\prime}$ and it is easily seen that $\sum_{\nu \in \mathbb{Z}}\left\langle\widetilde{\phi}_{\nu} * \phi_{\nu} * f_{s}, \eta\right\rangle=$ $\left\langle f_{s}, \eta\right\rangle$ for every $\eta \in \mathcal{S}_{0}(\mathbb{R})$, that is,

$$
\sum_{\nu \in \mathbb{Z}} \widetilde{\phi}_{\nu} * \phi_{\nu} * f_{s}=f_{s} \quad \text { in }\left(\mathcal{S}_{0}(\mathbb{R})\right)^{\prime} .
$$

Using the Hahn-Banach theorem, we can extend $\sum_{\nu \in \mathbb{Z}} \widetilde{\phi}_{\nu} * \phi_{\nu} * f_{s}$ from $\mathcal{S}_{0}(\mathbb{R})$ to a bounded linear functional on $\mathcal{S}(\mathbb{R})$, say $g \in(\mathcal{S}(\mathbb{R}))^{\prime}$, such that $\left.g\right|_{\mathcal{S}_{0}(\mathbb{R})}=$ $\left.f_{s}\right|_{\mathcal{S}_{0}(\mathbb{R})}$. The question that arises naturally is whether $f_{s}$ coincides with $g$ as elements of $(\mathcal{S}(\mathbb{R}))^{\prime}$. To address this, we shall construct such an extension $g$. For this we note that the convergence of the series $\sum_{\nu \in \mathbb{Z}} \widetilde{\phi}_{\nu} * \phi_{\nu} * f_{s}$ in $\left(\mathcal{S}_{0}(\mathbb{R})\right)^{\prime}$ is equivalent to the existence of constants $C_{N}$ and $C$ such that

$$
\lim _{N \rightarrow \infty}\left(\sum_{\nu=-N}^{\infty} \tilde{\phi}_{\nu} * \phi_{\nu} * f_{s}+C_{N}\right)=f_{s}+C
$$

in the sense of $(\mathcal{S}(\mathbb{R}))^{\prime}$. Thus, we can set

$$
g:=\lim _{N \rightarrow \infty}\left(\sum_{\nu=-N}^{\infty} \widetilde{\phi}_{\nu} * \phi_{\nu} * f_{s}+C_{N}\right) .
$$

One way of proving (2.7) (see also Lemma 6.2) is to define

$$
c_{\nu}:= \begin{cases}0, & \nu>0, \\ -\left\langle\left|\widehat{\phi}_{\nu}\right|^{2} \widehat{f}_{s}, \widehat{\theta}\right\rangle, & \nu \leq 0,\end{cases}
$$


where $\theta$ is the test function defined above. If $\eta \in \mathcal{S}(\mathbb{R})$, then

$$
\begin{aligned}
\lim _{N \rightarrow \infty} & \sum_{\nu=-N}^{\infty}\left\langle\widetilde{\phi}_{\nu} * \phi_{\nu} * f_{s}+c_{\nu}, \eta\right\rangle \\
= & \lim _{N \rightarrow \infty} \sum_{\nu=-N}^{\infty}\left(\left\langle\left(\widetilde{\phi}_{\nu} * \phi_{\nu} * f_{s}\right)^{\wedge}, \widehat{\eta}\right\rangle+c_{\nu} \widehat{\eta}(0)\right) \\
= & \lim _{N \rightarrow \infty} \sum_{\nu=-N}^{\infty}\left(\int_{0}^{\infty} \frac{\left|\widehat{\phi}_{\nu}(\xi)\right|^{2}}{\xi} \widehat{\eta}(\xi) d \xi+c_{\nu} \widehat{\eta}(0)\right) \\
= & \lim _{N \rightarrow \infty} \sum_{\nu=-N}^{\infty}\left(\int_{0}^{1} \frac{\left|\widehat{\phi}_{\nu}(\xi)\right|^{2}}{\xi} \widehat{\eta}(\xi) d \xi+c_{\nu} \widehat{\eta}(0)+\int_{1}^{\infty} \frac{\left|\widehat{\phi}_{\nu}(\xi)\right|^{2}}{\xi} \widehat{\eta}(\xi) d \xi\right) \\
= & \lim _{N \rightarrow \infty} \sum_{\nu=-N}^{0} \int_{0}^{1} \frac{\left|\widehat{\phi}_{\nu}(\xi)\right|^{2}}{\xi}(\widehat{\eta}(\xi)-\widehat{\eta}(0)) d \xi+\sum_{\nu=0}^{\infty} \int_{1}^{\infty} \frac{\left|\widehat{\phi}_{\nu}(\xi)\right|^{2}}{\xi} \widehat{\eta}(\xi) d \xi \\
= & \int_{0}^{1} \frac{(\widehat{\eta}(\xi)-\widehat{\eta}(0))}{\xi} d \xi+\int_{1}^{\infty} \frac{\widehat{\eta}(\xi)}{\xi} d \xi=\left\langle\widehat{f_{1}}, \widehat{\eta}\right\rangle=\left\langle f_{1}, \eta\right\rangle,
\end{aligned}
$$

where in the fifth equality we used Lebesgue's convergence theorem. This proves that

$$
\lim _{N \rightarrow \infty} \sum_{\nu=-N}^{\infty}\left(\widetilde{\phi}_{\nu} * \phi_{\nu} * f_{s}+c_{\nu}\right)=f_{1},
$$

and setting $C_{N}=\sum_{\nu=-N}^{0} c_{\nu}$ we get

$$
g:=\lim _{N \rightarrow \infty}\left(\sum_{\nu=-N}^{\infty} \widetilde{\phi}_{\nu} * \phi_{\nu} * f_{s}+C_{N}\right)=f_{1} .
$$

However, $f_{s}-f_{1}$ vanishes on $\mathcal{S}_{0}(\mathbb{R})$, and in particular from (2.5) it follows that for every $\eta \in \mathcal{S}(\mathbb{R})$,

$$
\left\langle\widehat{f}_{s}-\widehat{f_{1}}, \widehat{\eta}\right\rangle=-\ln (s) \widehat{\eta}(0)
$$

i.e.,

$$
f_{1}=f_{s}+\ln (s) \text {. }
$$

Putting together (2.9) and (2.10) we get

$$
g=f_{s}+\ln (s) \quad \text { in }(\mathcal{S}(\mathbb{R}))^{\prime} .
$$

Another way of writing (2.11) is

$$
\sum_{\nu \in \mathbb{Z}} \widetilde{\phi}_{\nu} * \phi_{\nu} * f_{s}=f_{s} \quad \text { in }(\mathcal{S}(\mathbb{R}))^{\prime} / \mathcal{P}_{0} .
$$


We note that (2.6) and (2.12) are equivalent formulations (see Lemma 6.2 for more details) and in this sense we write $\left(\mathcal{S}_{0}(\mathbb{R})\right)^{\prime} \approx(\mathcal{S}(\mathbb{R}))^{\prime} / \mathcal{P}_{0}$.

Since it is possible to construct similar examples with singularity of higher order at the origin, one is naturally led to the space $\mathcal{S}_{\infty}$ and for a general $f \in \mathcal{S}^{\prime}$ one has to consider the convergence of $\sum \widetilde{\phi}_{\nu} * \phi_{\nu} * f$ in the sense of $\left(\mathcal{S}_{\infty}\right)^{\prime}$. Using the continuity of the Fourier transform on $\mathcal{S}$ it is easy to see that $\mathcal{S}_{\infty}$ becomes a complete locally convex space (in the topology inherited from $\mathcal{S}$ ). It turns out (see [FJW]) that for every $f \in \mathcal{S}^{\prime}$,

$$
\sum_{\nu \in \mathbb{Z}} \widetilde{\phi}_{\nu} * \phi_{\nu} * f=f \quad \text { in }\left(\mathcal{S}_{\infty}\right)^{\prime} .
$$

A finer analysis, though, can be given in the cases where a distribution is in the class of Triebel-Lizorkin or Besov spaces. Let us assume that $f \in \dot{F}_{p q}^{s}$, $s \in \mathbb{R}, 0<p<\infty, 0<q \leq \infty$ (an identical analysis holds for the Besov spaces as well). Then, as was noted by Peetre [Pee] (see also [FJ2]), for every $\alpha \in \mathbb{N}^{d}$,

$$
\left\|\left(\widetilde{\phi}_{\nu} * \phi_{\nu} * f\right)^{(\alpha)}\right\|_{L_{\infty}} \leq C\left\|\widetilde{\phi}_{\nu}^{(\alpha)}\right\|_{L_{1}}\left\|\phi_{\nu} * f\right\|_{L_{\infty}} \leq C 2^{\nu(|\alpha|+d / p)}\left\|\phi_{\nu} * f\right\|_{L_{p}},
$$

where the second inequality follows from well known estimates on analytic functions (see [T, p. 22]). It follows that if $|\alpha|>s-d / p(|\alpha|=0$ for $d / p>s)$ then

$$
\sum_{\nu<0}\left\|\left(\widetilde{\phi}_{\nu} * \phi_{\nu} * f\right)^{(\alpha)}\right\|_{L_{\infty}} \leq C \sup _{\nu<0} 2^{\nu s}\left\|\phi_{\nu} * f\right\|_{L_{p}} \leq C\|f\|_{\dot{F}_{p q}^{s}}
$$

which implies that the series $\sum_{\nu \in \mathbb{Z}}\left(\widetilde{\phi}_{\nu} * \phi_{\nu} * f\right)^{(\alpha)}$ converges in $\mathcal{S}^{\prime}$ (if $s-d / p$ $\in \mathbb{N}$ and $0<q \leq 1$ in the $\dot{B}_{p q}^{s}$-case, or $0<p, q \leq 1$ in the $\dot{F}_{p q}^{s}$-case, we can take $|a|=s-d / p)$. Employing now Lemma 6.2 , for $k:=\max \{[s-d / p],-1\}$, we can find polynomials $P_{N} \in \mathcal{P}_{k}\left(\mathcal{P}_{-1}:=\{0\}\right)$ such that the series

$$
g:=\lim _{N \rightarrow \infty}\left(\sum_{\nu=-N}^{\infty} \widetilde{\phi}_{\nu} * \phi_{\nu} * f+P_{N}\right)
$$

converges in $\mathcal{S}^{\prime}$. On the other hand we see that $\operatorname{supp}(\widehat{g}-\widehat{f})=\{0\}$, i.e., there exists a polynomial $P \in \mathcal{P}$ such that

$$
g=f+P \quad \text { in } \mathcal{S}^{\prime} .
$$

In other words

$$
\sum_{\nu \in \mathbb{Z}} \widetilde{\phi}_{\nu} * \phi_{\nu} * f=f \quad \text { in } \mathcal{S}^{\prime} / \mathcal{P} .
$$

However, if we identify the equivalence class $f+\mathcal{P}$ with its representative $g$ then the elements of $\dot{F}_{p q}^{s}, \dot{B}_{p q}^{s}$ can be regarded as equivalence classes of distributions modulo polynomials in $\mathcal{P}_{k}$. Therefore, we may assume that 
$\dot{F}_{p q}^{s}, \dot{B}_{p q}^{s} \subset \mathcal{S}^{\prime} / \mathcal{P}_{k}\left(\mathcal{S}^{\prime} / \mathcal{P}_{-1}:=\mathcal{S}^{\prime}\right)$. Under this assumption for every $\eta \in \mathcal{S}_{k}$ we define

$$
\langle f, \eta\rangle:=\sum_{\nu \in \mathbb{Z}}\left\langle\widetilde{\phi}_{\nu} * \phi_{\nu} * f, \eta\right\rangle,
$$

i.e.,

$$
f=\sum_{\nu \in \mathbb{Z}} \widetilde{\phi}_{\nu} * \phi_{\nu} * f \quad \text { in } \mathcal{S}^{\prime} / \mathcal{P}_{k}
$$

It is an interesting question, given $f \in \dot{F}_{p q}^{s}$, to determine the degree of the polynomial $P$ in (2.13). It turns out (see Proposition 6.3) that if $f$ is of polynomial growth, that is, if

$$
\int_{\mathbb{R}^{d}}|f(x)|(1+|x|)^{-(d+k+1)} d x<\infty, \quad k \geq-1,
$$

then indeed $P \in \mathcal{P}_{k}$. Since $\int|f(x)|(1+|x|)^{-d} d x \leq C\|f\|_{L_{p}}, p>1$, this observation in particular identifies a distribution $f \in L_{p} \approx \dot{F}_{p 2}^{0}, 1<p<\infty$, with its representative $\sum_{\nu \in \mathbb{Z}} \widetilde{\phi}_{\nu} * \phi_{\nu} * f$.

More generally, in Proposition 6.3 we show that if $f \in \mathcal{S}^{\prime}$ satisfies

$$
|\langle f, \eta\rangle| \leq C \sup _{|\alpha| \leq N} \sup _{x \in \mathbb{R}^{d}}(1+|x|)^{k}\left|\eta^{(\alpha)}(x)\right|, \quad \eta \in \mathcal{S},
$$

for some $k, N \in \mathbb{N}$, then

$$
\sum_{\nu \in \mathbb{Z}} \widetilde{\phi}_{\nu} * \phi_{\nu} * f=f \quad \text { in } \mathcal{S}^{\prime} / \mathcal{P}_{k} .
$$

This is for instance the case if $f \in H_{p}, 0<p \leq 1$ (with $k=0, N>$ $d(1 / p-1))$.

The spaces $\dot{F}_{p q}^{s}$ and $\dot{B}_{p q}^{s}$ have been studied extensively, as we already mentioned, by Frazier and Jawerth in [FJ1], [FJ2]. Using techniques reminiscent of the Shannon sampling theorem, it was shown in [FJ1] that for every $f \in \mathcal{S}^{\prime}$,

$$
\widetilde{\phi}_{\nu} * \phi_{\nu} * f(x)=\sum_{I \in D_{\nu}}\left\langle f, \phi_{I}\right\rangle \phi_{I}(x), \quad x \in \mathbb{R}^{d},
$$

where $\phi_{I}(\cdot):=|I|^{-1 / 2} \phi\left(\left(\cdot-x_{I}\right) / \ell(I)\right)$. It follows that every distribution $f \in$ $\dot{F}_{p q}^{s}\left(\right.$ or $\left.\dot{B}_{p q}^{s}\right)$ can be represented in the form

$$
f=\sum_{\nu \in \mathbb{Z}} \sum_{I \in D_{\nu}}\left\langle f, \phi_{I}\right\rangle \phi_{I}=\sum_{I \in D}\left\langle f, \phi_{I}\right\rangle \phi_{I},
$$

where the outer summation is considered in the sense of $\mathcal{S}^{\prime} / \mathcal{P}_{k}$ with $k:=$ $\max \{[s-d / p],-1\}$. This is the so-called $\phi$-transform identity.

Moreover, the coefficients

$$
s_{I}(f):=\left\langle f, \phi_{I}\right\rangle, \quad I \in D,
$$


in (2.16) contain all the necessary information to determine whether a distribution belongs in the class of Triebel-Lizorkin or Besov spaces. In particular, it was established in [FJ2] that if $s \in \mathbb{R}, 0<p<\infty$ and $0<q \leq \infty$ then

$$
\|f\|_{\dot{F}_{p q}^{s}} \approx\left\|\left(\sum_{I \in D}\left(|I|^{-s / d}\left|s_{I}(f)\right| \tilde{\chi}_{I}\right)^{q}\right)^{1 / q}\right\|_{p}
$$

where as before $\widetilde{\chi}_{I}:=|I|^{-1 / 2} \chi_{I}$.

Similarly if $s \in \mathbb{R}$ and $0<p, q \leq \infty$ (see [FJ1]) then

$$
\|f\|_{\dot{B}_{p q}^{s}} \approx\left(\sum_{m \in \mathbb{Z}}\left(\sum_{I \in D_{m}}\left(|I|^{-s / d+(1 / p-1 / 2)}\left|s_{I}(f)\right|\right)^{p}\right)^{q / p}\right)^{1 / q} .
$$

3. Bounded operators on sequence spaces. In order to prove that a decomposition system for $L_{2}\left(\mathbb{R}^{d}\right)$ constitutes a decomposition system for the spaces $\dot{F}_{p q}^{s}$ and $\dot{B}_{p q}^{s}$, we will use the boundedness of operators on the corresponding sequence spaces $\dot{f}_{p q}^{s}$ and $\dot{b}_{p q}^{s}$. As already mentioned, this type of questions, regarding the $\dot{f}_{p q}^{s}$ spaces, have been thoroughly studied in [FJ2]. In particular, a proof of Proposition 3.1, based on duality arguments, can be found in [FJ2]. Nevertheless, for the sake of completeness we present here a straightforward approach.

We start by recalling the definition of the $\dot{f}_{p q}^{s}$ and $\dot{b}_{p q}^{s}$ spaces.

(a) For $s \in \mathbb{R}, 0<p<\infty$, and $0<q \leq \infty$, $\dot{f}_{p q}^{s}$ is defined to be the space of all sequences $h:=\left(h_{I}\right)_{I \in D}$ such that

$$
\|h\|_{\dot{f}_{p q}^{s}}:=\left\|\left(\sum_{I \in D}\left(|I|^{-s / d}\left|h_{I}\right| \widetilde{\chi}_{I}\right)^{q}\right)^{1 / q}\right\|_{L_{p}}<\infty .
$$

(b) For $s \in \mathbb{R}$ and $0<p, q \leq \infty, \dot{b}_{p q}^{s}$ is defined to be the space of all sequences $h:=\left(h_{I}\right)_{I \in D}$ such that

$$
\|h\|_{\dot{b}_{p q}^{s}}:=\left(\sum_{m \in \mathbb{Z}}\left(\sum_{I \in D_{m}}\left(|I|^{-s / d+1 / p-1 / 2}\left|h_{I}\right|\right)^{p}\right)^{q / p}\right)^{1 / q}<\infty .
$$

Since the family $B=\left\{\theta_{I}^{e}, \widetilde{\theta}_{I}^{e}: I \in D, e \in E\right\}$ forms a decomposition system for $L_{2}\left(\mathbb{R}^{d}\right)$, for every $I \in D$ we have

$$
\phi_{I}=\sum_{e \in E} \sum_{J \in D} \widetilde{a}_{e}(I, J) \theta_{J}^{e}, \quad \widetilde{a}_{e}(I, J):=\left\langle\phi_{I}, \widetilde{\theta}_{J}^{e}\right\rangle .
$$

Similarly from (2.16), for every $e \in E, I \in D$ we have

$$
\theta_{I}^{e}=\sum_{J \in D} a_{e}(I, J) \phi_{J}, \quad a_{e}(I, J):=\left\langle\theta_{I}^{e}, \phi_{J}\right\rangle .
$$

In essence our results are based on the fact that the transpose of the transformation matrices of one decomposition system with respect to the 
other,

$$
\widetilde{\mathbf{A}}_{e}:=\left(\widetilde{a}_{e}(I, J)\right)_{I, J \in D}, \quad \mathbf{A}_{e}:=\left(a_{e}(I, J)\right)_{I, J \in D},
$$

are very close to the identity matrix, and thus, as we shall prove, are bounded on $\dot{f}_{p q}^{s}$ and $\dot{b}_{p q}^{s}$ for appropriate range of the indices $s, p, q$.

In order to measure the size of the entries of the matrices $\widetilde{\mathbf{A}}_{e}, \mathbf{A}_{e}, e \in E$, we shall need the following lemma:

Lemma 3.1. Let $I, J \in D$ with $|J| \leq|I|$. Assume moreover that $\eta_{J}, \theta_{I}$ are functions on $\mathbb{R}^{d}$ such that for some $r \geq 1$ and $M>d+r$,

$$
\begin{gathered}
\int_{\mathbb{R}^{d}} x^{\alpha} \eta_{J}(x) d x=0, \quad|\alpha| \leq r-1, \\
\left|\eta_{J}(x)\right| \leq C|J|^{-1 / 2}\left(1+\frac{\left|x-x_{J}\right|}{\ell(J)}\right)^{-M}, \\
\left|\left(\theta_{I}\right)^{(\alpha)}(x)\right| \leq C|I|^{-1 / 2-|\alpha| / d}\left(1+\frac{\left|x-x_{I}\right|}{\ell(I)}\right)^{-M}, \quad|\alpha| \leq r .
\end{gathered}
$$

Then

$$
\left|\left\langle\theta_{I}, \eta_{J}\right\rangle\right| \leq C\left(\frac{\ell(J)}{\ell(I)}\right)^{r+d / 2}\left(1+\frac{\left|x_{I}-x_{J}\right|}{\ell(I)}\right)^{-M} .
$$

Proof. We refer the reader to [FJ2].

REMARK 3.1. In the absence of zero moments, that is, if $|J| \leq|I|$ and

$$
\begin{gathered}
\left|\eta_{J}(x)\right| \leq C|J|^{-1 / 2}\left(1+\frac{\left|x-x_{J}\right|}{\ell(J)}\right)^{-M}, \\
\left|\theta_{I}(x)\right| \leq C|I|^{-1 / 2}\left(1+\frac{\left|x-x_{I}\right|}{\ell(I)}\right)^{-M},
\end{gathered}
$$

with $M>d$, using arguments similar to the proof of the previous lemma it is easy to show that

$$
\left|\left\langle\theta_{I}, \eta_{J}\right\rangle\right| \leq C\left(\frac{\ell(J)}{\ell(I)}\right)^{d / 2}\left(1+\frac{\left|x_{I}-x_{J}\right|}{\ell(I)}\right)^{-M} .
$$

REMARK 3.2. It is obvious from the above lemma that the size of $\left|\left\langle\theta_{I}, \eta_{J}\right\rangle\right|$, when $|J| \leq|I|$, depends on the smoothness of $\theta_{I}$ and the zero moments of $\eta_{J}$, while in the case where $|J|>|I|$ the requirements on $\theta_{I}, \eta_{J}$ have to be interchanged. For this reason for the rest of the section we shall assume that for some $r_{1}, r_{2} \in \mathbb{N}$ and $M>d+\max \left\{r_{1}, r_{2}\right\},\left(\theta_{I}\right)_{I \in D},\left(\eta_{I}\right)_{I \in D}$ are families of functions on $\mathbb{R}^{d}$ satisfying

$$
\int_{\mathbb{R}^{d}} \theta_{I}(x) x^{\alpha} d x=0, \quad|\alpha| \leq r_{1}-1,
$$




$$
\begin{aligned}
\left|\theta_{I}^{(\alpha)}(x)\right| \leq & C|I|^{-1 / 2-\alpha / d}\left(1+\frac{\left|x-x_{I}\right|}{\ell(I)}\right)^{-M}, \quad|\alpha| \leq r_{2}, \\
& \int_{\mathbb{R}^{d}} \eta_{I}(x) x^{\alpha} d x=0, \quad|\alpha| \leq r_{2}-1, \\
\left|\eta_{I}^{(\alpha)}(x)\right| \leq & C|I|^{-1 / 2-\alpha / d}\left(1+\frac{\left|x-x_{I}\right|}{\ell(I)}\right)^{-M}, \quad|\alpha| \leq r_{1},
\end{aligned}
$$

where (3.5) and (3.7) are void when $r_{1}=0$ or $r_{2}=0$.

Assuming that $r_{1}, r_{2}$ and $M$ are sufficiently large we shall prove that the infinite matrix

$$
\mathbf{A}:=\left(\left\langle\theta_{I}, \eta_{J}\right\rangle\right)_{I, J \in D}
$$

gives rise to a bounded operator on the $\dot{f}$ and $\dot{b}$ spaces.

Proposition 3.1. Let $\left(\theta_{I}\right)_{I \in D},\left(\eta_{I}\right)_{I \in D}$ be families of functions satisfying (3.5)-(3.8) for some $r_{1}, r_{2} \in \mathbb{N}$ and $M>d+\max \left\{r_{1}, r_{2}\right\}$. Let also $0<p<\infty, 0<q \leq \infty, s \in \mathbb{R}$ and $\mathcal{J}:=d / \min \{1, p, q\}$. If $r_{1}>s$, $r_{2}>\mathcal{J}-d-s$ and $M>\mathcal{J}$ then the matrix $\mathbf{A}$ in (3.9) defines a bounded operator on $\dot{f}_{p q}^{s}$.

Proof. For $\mathbf{A}$ to be bounded on $\dot{f}_{p q}^{s}$ it is sufficient to prove that

$$
\|\mathbf{A}\|_{\dot{f}_{p q}^{s} \rightarrow \dot{f}_{p q}^{s}}:=\sup _{\|h\|_{f_{p q}^{s}} \leq 1}\|\mathbf{A} h\|_{\dot{f}_{p q}^{s}}<\infty .
$$

Let $a(I, J):=\left\langle\theta_{I}, \eta_{J}\right\rangle$. Then for every $h \in \dot{f}_{p q}^{s}$,

$$
(\mathbf{A} h)_{I}=\sum_{J \in D} a(I, J) h_{J}
$$

where the series is absolutely convergent (see proof below). It follows that

$$
\begin{aligned}
\|\mathbf{A} h\|_{\dot{f}_{p q}^{s}} & =\left\|\left(\sum_{I \in D}\left(|I|^{-s / d}\left|(\mathbf{A} h)_{I}\right| \widetilde{\chi}_{I}\right)^{q}\right)^{1 / q}\right\|_{L_{p}} \\
& \leq\left\|\left(\sum_{I \in D}\left(|I|^{-s / d} \sum_{J \in D}|a(I, J)|\left|h_{J}\right| \widetilde{\chi}_{I}\right)^{q}\right)^{1 / q}\right\|_{L_{p}} \\
& \leq C\left(\sigma_{1}+\sigma_{2}\right),
\end{aligned}
$$

where

$$
\begin{aligned}
& \sigma_{1}:=\left\|\left(\sum_{I \in D}\left(|I|^{-s / d} \sum_{|J| \leq|I|}|a(I, J)|\left|h_{J}\right| \tilde{\chi}_{I}\right)^{q}\right)^{1 / q}\right\|_{L_{p}}, \\
& \sigma_{2}:=\left\|\left(\sum_{I \in D}\left(|I|^{-s / d} \sum_{|J|>|I|}|a(I, J)|\left|h_{J}\right| \tilde{\chi}_{I}\right)^{q}\right)^{1 / q}\right\|_{L_{p}} .
\end{aligned}
$$


To estimate $\sigma_{1}$, from Lemma 3.1 (or Remark 3.1 for $r_{2}=0$ ), if $|J| \leq|I|$ then

$$
|a(I, J)| \leq C\left(\frac{\ell(J)}{\ell(I)}\right)^{r_{2}+d / 2}\left(1+\frac{\left|x_{I}-x_{J}\right|}{\ell(I)}\right)^{-M}
$$

Let $\lambda_{I}:=|I|^{-s / d} \widetilde{\chi}_{I}$ and $0<t<\min \{1, p, q\}$ be such that $M>d / t$ and $r_{2}>d(1 / t-1)-s$. Using Lemmas 7.1 and 7.2 we obtain

$$
\begin{aligned}
& \sigma_{1} \leq C\left\|\left(\sum_{I \in D}\left(\sum_{|J| \leq|I|}\left(\frac{\ell(J)}{\ell(I)}\right)^{r_{2}+d / 2}\left(1+\frac{\left|x_{I}-x_{J}\right|}{\ell(I)}\right)^{-M}\left|h_{J}\right| \lambda_{I}\right)^{q}\right)^{1 / q}\right\|_{L_{p}} \\
& =C\left\|\left(\sum_{n \in \mathbb{Z}} \sum_{I \in D_{n}}\left(\sum_{m \geq n} 2^{(n-m)\left(r_{2}+d / 2\right)} \sum_{J \in D_{m}}\left(1+\frac{\left|x_{I}-x_{J}\right|}{\ell(I)}\right)^{-M}\left|h_{J}\right| \lambda_{I}\right)^{q}\right)^{1 / q}\right\|_{L_{p}} \\
& \leq C\left\|\left(\sum_{n \in \mathbb{Z}} \sum_{I \in D_{n}}\left(\sum_{m \geq n} 2^{(n-m)\left(r_{2}+d / 2-d / t\right)} M_{t}\left(\sum_{J \in D_{m}}\left|h_{J}\right| \chi_{J}\right)(x) \lambda_{I}(x)\right)^{q}\right)^{1 / q}\right\|_{L_{p}} \\
& =C\left\|\left(\sum_{n \in \mathbb{Z}}\left(\sum_{m \geq n} 2^{(n-m)\left(r_{2}+d-d / t+s\right)} M_{t}\left(\sum_{J \in D_{m}}\left|h_{J}\right| \lambda_{J}\right)(x)\right)^{q}\right)^{1 / q}\right\| L_{L_{p}} \\
& \leq C\left\|\left(\sum_{n \in \mathbb{Z}}\left(M_{t}\left(\sum_{I \in D_{n}}\left|h_{I}\right| \lambda_{I}\right)(x)\right)^{q}\right)^{1 / q}\right\|\left\|_{L_{p}} \leq C\right\| h \|_{\dot{f}_{p q}^{s}}
\end{aligned}
$$

where in the last inequality we used (7.1) $(t<\min \{1, p, q\})$.

When $|J|>|I|$, by interchanging the roles of $\eta_{J}$ and $\theta_{I}$ in Lemma 3.1 (or in Remark 3.1 for $r_{1}=0$ ), we have

$$
|a(I, J)| \leq C\left(\frac{\ell(I)}{\ell(J)}\right)^{r_{1}+d / 2}\left(1+\frac{\left|x_{I}-x_{J}\right|}{\ell(J)}\right)^{-M}
$$

Employing Lemmas 7.2 and 7.1 gives

$$
\begin{aligned}
& \sigma_{2} \leq C\left\|\left(\sum_{I \in D}\left(\sum_{|J|>|I|}\left(\frac{\ell(I)}{\ell(J)}\right)^{r_{1}+d / 2}\left(1+\frac{\left|x_{I}-x_{J}\right|}{\ell(J)}\right)^{-M}\left|h_{J}\right| \lambda_{I}\right)^{q}\right)^{1 / q}\right\|_{L_{p}} \\
& =C\left\|\left(\sum_{n \in \mathbb{Z}} \sum_{I \in D_{n}}\left(\sum_{m<n} 2^{(m-n)\left(r_{1}+d / 2\right)} \sum_{J \in D_{m}}\left(1+\frac{\left|x_{I}-x_{J}\right|}{\ell(J)}\right)^{-M}\left|h_{J}\right| \lambda_{I}\right)^{q}\right)^{1 / q}\right\|_{L_{p}} \\
& \leq C\left\|\left(\sum_{n \in \mathbb{Z}} \sum_{I \in D_{n}}\left(\sum_{m<n} 2^{(m-n)\left(r_{1}+d / 2\right)} M_{t}\left(\sum_{J \in D_{m}}\left|h_{J}\right| \chi_{J}\right)(x) \lambda_{I}(x)\right)^{q}\right)^{1 / q}\right\|_{L_{p}} \\
& =C\left\|\left(\sum_{n \in \mathbb{Z}}\left(\sum_{m<n} 2^{(m-n)\left(r_{1}-s\right)} M_{t}\left(\sum_{J \in D_{m}}\left|h_{J}\right| \lambda_{J}\right)(x)\right)^{q}\right)^{1 / q}\right\|_{L_{p}} \\
& \leq C\left\|\left(\sum_{n \in \mathbb{Z}}\left(M_{t}\left(\sum_{I \in D_{n}}\left|h_{I}\right| \lambda_{I}\right)(x)\right)^{q}\right)^{1 / q}\right\|\left\|_{L_{p}} \leq C\right\| h \|_{\dot{f}_{p q}^{s}},
\end{aligned}
$$


where in the fourth inequality we used the inequality $r_{1}>s$. Employing the two estimates for $\sigma_{1}$ and $\sigma_{2}$ in (3.10) yields the result.

Turning now our attention to the Besov spaces we have the following:

Proposition 3.2. Let $\left(\theta_{I}\right)_{I \in D},\left(\eta_{I}\right)_{I \in D}$ be families of functions satisfying (3.5)-(3.8) for some $r_{1}, r_{2} \in \mathbb{N}$ and $M>d+\max \left\{r_{1}, r_{2}\right\}$. Assume moreover that $0<p, q \leq \infty, s \in \mathbb{R}$ and $\mathcal{J}:=d / \min \{1, p\}$. If $r_{1}>s$, $r_{2}>\mathcal{J}-d-s$ and $M>\mathcal{J}$ then the matrix $\mathbf{A}$ in (3.9) defines a bounded operator on $\dot{b}_{p q}^{s}$.

Proof. We shall treat only the cases where $0<p, q<\infty$; when $p=\infty$ or $q=\infty$ the result follows similarly. We need to prove that

$$
\|\mathbf{A}\|_{\dot{p}_{p q}^{s} \rightarrow \dot{b}_{p q}^{s}}:=\sup _{\|h\|_{\dot{b}_{p q}^{s}} \leq 1}\|\mathbf{A} h\|_{\dot{b}_{p q}^{s}}<\infty
$$

Let $a(I, J):=\left\langle\theta_{I}, \eta_{J}\right\rangle$ and $h \in \dot{b}_{p q}^{s}$. To simplify our notation we define $\gamma:=s / d-(1 / p-1 / 2)$ and $\widetilde{h}_{J}:=|J|^{-\gamma} h_{J}$. Since $(\mathbf{A} h)_{I}=\sum_{J \in D} a(I, J) h_{J}$,

$$
\begin{aligned}
\|\mathbf{A} h\|_{\dot{b}_{p q}^{s}} & =\sum_{m \in \mathbb{Z}}\left(\sum_{I \in D_{m}}\left(|I|^{-\gamma}\left|(\mathbf{A} h)_{I}\right|\right)^{p}\right)^{q / p} \\
& \leq \sum_{m \in \mathbb{Z}}\left(\sum_{I \in D_{m}}\left(\sum_{J \in D}(|J| /|I|)^{\gamma}|a(I, J)| \widetilde{h}_{J} \mid\right)^{p}\right)^{q / p} \leq C\left(\sigma_{1}^{q}+\sigma_{2}^{q}\right),
\end{aligned}
$$

with

$$
\begin{aligned}
\sigma_{1} & :=\left(\sum_{m \in \mathbb{Z}}\left(\sum_{I \in D_{m}}\left(\sum_{|J| \leq|I|}(|J| /|I|)^{\gamma}|a(I, J)|\left|\widetilde{h}_{J}\right|\right)^{p}\right)^{q / p}\right)^{1 / q}, \\
\sigma_{2} & :=\left(\sum_{m \in \mathbb{Z}}\left(\sum_{I \in D_{m}}\left(\sum_{|J|>|I|}(|J| /|I|)^{\gamma}|a(I, J)|\left|\widetilde{h}_{J}\right|\right)^{p}\right)^{q / p}\right)^{1 / q} .
\end{aligned}
$$

CASE I: $1 \leq p<\infty$. For $\sigma_{1}$ using (3.11) (or (3.4) for $r_{2}=0$ ), Minkowski's inequality and Lemmas 7.2 and 7.4 we get

$$
\begin{aligned}
\sigma_{1}^{q} \leq & \sum_{m \in \mathbb{Z}}\left(\sum_{I \in D_{m}}\left(\sum_{n \geq m} \sum_{J \in D_{n}}(|J| /|I|)^{\gamma}|a(I, J)|\left|\widetilde{h}_{J}\right|\right)^{p}\right)^{q / p} \\
\leq & \sum_{m \in \mathbb{Z}}\left(\sum_{I \in D_{m}}\left(\sum_{n \geq m} \sum_{J \in D_{n}}(|J| /|I|)^{\gamma+r_{2} / d+1 / 2}\left(1+\frac{\left|x_{I}-x_{J}\right|}{\ell(I)}\right)^{-M}\left|\widetilde{h}_{J}\right|\right)^{p}\right)^{q / p} \\
\leq & C \sum_{m \in \mathbb{Z}}\left(\sum_{n \geq m} 2^{(m-n)\left(d \gamma+r_{2}+d / 2\right)}\right. \\
& \left.\times\left(\sum_{I \in D_{m}}\left(\sum_{J \in D_{n}}\left(1+\frac{\left|x_{I}-x_{J}\right|}{\ell(I)}\right)^{-M}\left|\widetilde{h}_{J}\right|\right)^{p}\right)^{1 / p}\right)^{q}
\end{aligned}
$$




$$
\begin{aligned}
& \leq C \sum_{m \in \mathbb{Z}}\left(\sum_{n \geq m} 2^{(m-n)\left(d \gamma+r_{2}-d / 2+d / p\right)}\left(\sum_{J \in D_{n}}\left|\widetilde{h}_{J}\right|^{p}\right)^{1 / p}\right)^{q} \\
& \leq C \sum_{m \in \mathbb{Z}}\left(\sum_{I \in D_{m}}\left(|I|^{-s / d+(1 / p-1 / 2)}\left|h_{I}\right|\right)^{p}\right)^{q / p}
\end{aligned}
$$

since $d \gamma+r_{2}-d / 2+d / p=r_{2}+s>0$.

Similarly, using (3.12) (or (3.4) for $r_{1}=0$ ), Minkowski's inequality and Lemma 7.5 we obtain

$$
\begin{aligned}
& \sigma_{2}^{q} \leq \sum_{m \in \mathbb{Z}}\left(\sum_{I \in D_{m}}\left(\sum_{n<m} \sum_{J \in D_{n}}(|J| /|I|)^{\gamma}|a(I, J)|\left|\tilde{h}_{J}\right|\right)^{p}\right)^{q / p} \\
& \leq \sum_{m \in \mathbb{Z}}\left(\sum_{I \in D_{m}}\left(\sum_{n<m} \sum_{J \in D_{n}}(|J| /|I|)^{\gamma-r_{1} / d-1 / 2}\left(1+\frac{\left|x_{I}-x_{J}\right|}{\ell(J)}\right)^{-M}\left|\widetilde{h}_{J}\right|\right)^{p}\right)^{q / p} \\
& \leq C \sum_{m \in \mathbb{Z}}\left(\sum_{n<m} 2^{(m-n)\left(d \gamma-r_{1}-d / 2\right)}\right. \\
& \left.\times\left(\sum_{I \in D_{m}}\left(\sum_{J \in D_{n}}\left(1+\frac{\left|x_{I}-x_{J}\right|}{\ell(J)}\right)^{-M}\left|\tilde{h}_{J}\right|\right)^{p}\right)^{1 / p}\right)^{q} \\
& \leq C \sum_{m \in \mathbb{Z}}\left(\sum_{n<m} 2^{(m-n)\left(d \gamma-r_{1}-d / 2+d / p\right)}\left(\sum_{J \in D_{n}}\left|\widetilde{h}_{J}\right|^{p}\right)^{1 / p}\right)^{q} \\
& \leq C \sum_{m \in \mathbb{Z}}\left(\sum_{I \in D_{m}}\left(|I|^{-s / d+(1 / p-1 / 2)}\left|h_{I}\right|\right)^{p}\right)^{q / p},
\end{aligned}
$$

where in the last inequality we applied Lemma $7.2\left(d \gamma-r_{1}-d / 2+d / p=\right.$ $\left.s-r_{1}<0\right)$. Putting the estimates for $\sigma_{1}$ and $\sigma_{2}$ together we find that $(3.13)$ holds for $1 \leq p<\infty$.

CASE II: $p \leq 1$. Similarly to the previous case,

$$
\begin{aligned}
\sigma_{1}^{q} & \leq C \sum_{m \in \mathbb{Z}}\left(\sum_{J \in D_{n}} \sum_{n \geq m} \sum_{I \in D_{m}} 2^{(m-n)\left(r_{2}+d / 2+\gamma d\right) p}\left(1+\frac{\left|x_{I}-x_{J}\right|}{\ell(I)}\right)^{-M p}\left|\widetilde{h}_{J}\right|^{p}\right)^{q / p} \\
& \leq C \sum_{m \in \mathbb{Z}}\left(\sum_{J \in D_{n}} \sum_{n \geq m} 2^{(m-n)\left(r_{2}+d / 2+\gamma d\right) p}\left|\widetilde{h}_{J}\right|^{p}\right)^{q / p} \\
& =C \sum_{m \in \mathbb{Z}}\left(\sum_{n \geq m} 2^{(m-n)\left(r_{2}+s-d(1 / p-1)\right) p} \sum_{J \in D_{n}}\left|\widetilde{h}_{J}\right|^{p}\right)^{q / p} \\
& \leq C \sum_{m \in \mathbb{Z}}\left(\sum _ { I \in D _ { m } } \left(|I|^{\left.\left.-s / d+(1 / p-1 / 2)\left|h_{I}\right|\right)^{p}\right)^{q / p}}\right.\right.
\end{aligned}
$$

where in the last inequality we used the inequality $r_{2}>\mathcal{J}-d-s$. 
Finally, from Lemmas 7.2 and 7.3,

$$
\begin{aligned}
\sigma_{2}^{q} & \leq C \sum_{m \in \mathbb{Z}}\left(\sum_{n<m} \sum_{J \in D_{n}} \sum_{I \in D_{m}} 2^{(n-m)\left(r_{1}+d / 2-\gamma d\right) p}\left(1+\frac{\left|x_{I}-x_{J}\right|}{\ell(J)}\right)^{-M p}\left|\widetilde{h}_{J}\right|^{p}\right)^{q / p} \\
& \leq C \sum_{m \in \mathbb{Z}}\left(\sum_{n<m} \sum_{J \in D_{n}} 2^{(n-m)\left(r_{1}+d / 2-\gamma d\right) p} 2^{(m-n) d}\left|\widetilde{h}_{J}\right|^{p}\right)^{q / p} \\
& =C \sum_{m \in \mathbb{Z}}\left(\sum_{n<m} 2^{(n-m)\left(r_{1}-s\right) p} \sum_{J \in D_{n}}\left|\widetilde{h}_{J}\right|^{p}\right)^{q / p} \\
& \leq C \sum_{m \in \mathbb{Z}}\left(\sum_{I \in D_{m}}\left(|I|^{-s / d+(1 / p-1 / 2)}\left|h_{I}\right|\right)^{p}\right)^{q / p} .
\end{aligned}
$$

This concludes the proof of (3.13).

4. Main results. In this section we shall establish our main characterizations of the Triebel-Lizorkin and Besov spaces. We recall from the introduction that $\mathcal{S}_{-1}:=\mathcal{S}$ and that $\mathcal{S}^{\prime} / \mathcal{P}_{-1}:=\mathcal{S}^{\prime}$.

Theorem 4.1. Let $s \in \mathbb{R}, 0<p<\infty, 0<q \leq \infty, \mathcal{J}:=d / \min \{1, p, q\}$ and $k=\max \{[s-d / p],-1\}$. Let also $\theta_{I}^{e}, \widetilde{\theta}_{I}^{e}, I \in D, e \in E$, be a decomposition system for $L_{2}\left(\mathbb{R}^{d}\right)$ satisfying (1.1) and (1.2) for some $r_{\Theta}, r_{\widetilde{\Theta}} \in \mathbb{N}$ with $r_{\Theta}$ $>s, r_{\widetilde{\Theta}}>\mathcal{J}-d-s$ and $M_{\Theta}, M_{\widetilde{\Theta}}>\max \left\{\mathcal{J}, d+r_{\Theta}, d+r_{\tilde{\Theta}}\right\}$. Then, for every $f \in \dot{F}_{p q}^{s}$,

$$
f=\sum_{e \in E} \sum_{I \in D} \widetilde{a}_{I}^{e}(f) \theta_{I}^{e}, \quad \text { where } \widetilde{a}_{I}^{e}(f)=\left\langle f, \widetilde{\theta}_{I}^{e}\right\rangle,
$$

in the sense of $\mathcal{S}^{\prime} / \mathcal{P}_{k}$ (and in $\dot{F}_{p q}^{s}$ for $\left.q \neq \infty\right)$. Moreover,

$$
\|f\|_{\dot{F}_{p q}^{s}} \approx \sum_{e \in E}\left\|\left(\widetilde{a}_{I}^{e}(f)\right)_{I}\right\|_{\dot{f}_{p q}^{s}} .
$$

Adapting the above theorem to the case of biorthogonal wavelet bases for $L_{2}\left(\mathbb{R}^{d}\right)$ we immediately get

Proposition 4.1. Let $s \in \mathbb{R}, 0<p<\infty, 0<q \leq \infty, \mathcal{J}:=d / \min \{1, p, q\}$ and $k=\max \{[s-d / p],-1\}$. Let also $\Psi, \widetilde{\Psi}$ be dual wavelet sets for $L_{2}\left(\mathbb{R}^{d}\right)$ satisfying (1.13) and (1.14) for some $r_{\Psi}, r_{\widetilde{\Psi}} \in \mathbb{N}_{+}$with $r_{\Psi}>s, r_{\widetilde{\Psi}}>\mathcal{J}-d-s$ and $M_{\Psi}, M_{\widetilde{\Psi}}>\max \left\{\mathcal{J}, d+r_{\Psi}, d+r_{\widetilde{\Psi}}\right\}$. Then, for every $f \in \dot{F}_{p q}^{s}$, there exist unique coefficients

$$
\widetilde{c}_{I}^{e}(f):=\left\langle f, \widetilde{\psi}_{I}^{e}\right\rangle, \quad e \in E, I \in D,
$$

such that

$$
f=\sum_{e \in E} \sum_{I \in D} \widetilde{c}_{I}^{e}(f) \psi_{I}^{e}
$$


in the sense of $\mathcal{S}^{\prime} / \mathcal{P}_{k}$ (and in $\dot{F}_{p q}^{s}$ for $\left.q \neq \infty\right)$. Moreover,

$$
\|f\|_{\dot{F}_{p q}^{s}} \approx \sum_{e \in E}\left\|\left(\widetilde{c}_{I}^{e}(f)\right)_{I}\right\|_{\dot{f}_{p q}^{s}} .
$$

As far as the Besov spaces are concerned we shall prove:

Theorem 4.2. Let $s \in \mathbb{R}, 0<p, q \leq \infty, \mathcal{J}:=d / \min \{1, p\}$ and $k=$ $\max \{[s-d / p],-1\}$. Let also $\theta_{I}^{e}, \widetilde{\theta}_{I}^{e}, I \in D, e \in E$, be a decomposition system for $L_{2}\left(\mathbb{R}^{d}\right)$ satisfying (1.1) and (1.2) for some $r_{\Theta}, r_{\tilde{\Theta}} \in \mathbb{N}$ with $r_{\Theta}>s$, $r_{\tilde{\Theta}}>\mathcal{J}-d-s$ and $M_{\Theta}, M_{\widetilde{\Theta}}>\max \left\{\mathcal{J}, d+r_{\Theta}, d+r_{\tilde{\Theta}}\right\}$. Then, for every $f \in \dot{B}_{p q}^{s}$,

$$
f=\sum_{e \in E} \sum_{I \in D} \widetilde{a}_{I}^{e}(f) \theta_{I}^{e}, \quad \text { where } \widetilde{a}_{I}^{e}(f)=\left\langle f, \widetilde{\theta}_{I}^{e}\right\rangle
$$

in the sense of $\mathcal{S}^{\prime} / \mathcal{P}_{k}$ (and in $\dot{B}_{p q}^{s}$ for $p, q \neq \infty$ ). Moreover,

$$
\|f\|_{\dot{B}_{p q}^{s}} \approx \sum_{e \in E}\left\|\left(\widetilde{a}_{I}^{e}(f)\right)_{I}\right\|_{\dot{b}_{p q}^{s}} .
$$

Applying this theorem to biorthogonal wavelet bases we get

Proposition 4.2. Let $s \in \mathbb{R}, 0<p, q \leq \infty, \mathcal{J}:=d / \min \{1, p\}$ and $k=$ $\max \{[s-d / p],-1\}$. Let also $\Psi, \widetilde{\Psi}$ be dual wavelet sets for $L_{2}\left(\mathbb{R}^{d}\right)$ satisfying (1.13) and (1.14) for some $r_{\Psi}, r_{\widetilde{\Psi}} \in \mathbb{N}_{+}$with $r_{\Psi}>s, r_{\widetilde{\Psi}}>\mathcal{J}-d-s$ and $M_{\Psi}, M_{\widetilde{\Psi}}>\max \left\{\mathcal{J}, d+r_{\Psi}, d+r_{\widetilde{\Psi}}\right\}$. Then, for every $f \in \dot{B}_{p q}^{s}$, there exist unique coefficients $\widetilde{c}_{I}^{e}(f), e \in E, I \in D$, given by (4.3) such that

$$
f=\sum_{e \in E} \sum_{I \in D} \widetilde{c}_{I}^{e}(f) \psi_{I}^{e}
$$

in the sense of $\mathcal{S}^{\prime} / \mathcal{P}_{k}$ (and in $\dot{B}_{p q}^{s}$ for $\left.p, q \neq \infty\right)$. Moreover,

$$
\|f\|_{\dot{B}_{p q}^{s}} \approx \sum_{e \in E}\left\|\left(\widetilde{c}_{I}^{e}(f)\right)_{I}\right\|_{\dot{b}_{p q}^{s}} .
$$

For the proofs of Theorems 4.1, 4.2, we shall need the following lemmas:

Lemma 4.1. Let $s \in \mathbb{R}, 0<p<\infty, 0<q \leq \infty, \mathcal{J}:=d / \min \{1, p, q\}$ and $k=\max \{[s-d / p],-1\}$. If $\left(\theta_{I}\right)_{I \in D}$ satisfies (3.5)-(3.6) for some $r_{1}, r_{2} \in \mathbb{N}$ with $r_{1}>\mathcal{J}-d-s, r_{2}>s$ and $M>\max \left\{\mathcal{J}, d+r_{1}, d+r_{2}\right\}$, then for every $d:=\left(d_{I}\right) \in \dot{f}_{p q}^{s}$ the series $\sum_{I \in D} d_{I} \theta_{I}$ converges in $\mathcal{S}^{\prime} / \mathcal{P}_{k}$ (and in $\dot{F}_{p q}^{s}$ for $q \neq \infty)$ and

$$
\left\|\sum_{I \in D} d_{I} \theta_{I}\right\|_{\dot{F}_{p q}^{s}} \leq C\|d\|_{\dot{f}_{p q}^{s}}
$$

Proof. Let $\eta \in S_{k}$. Employing Lemma 3.1 (or Remark 3.1 for $k=-1$ or $r_{1}=0$ ), we find that for $|I|>1$, 


$$
\left|\left\langle\theta_{I}, \eta\right\rangle\right| \leq C \ell(I)^{-(k+1+d / 2)}\left(1+\frac{\left|x_{I}\right|}{\ell(I)}\right)^{-M},
$$

while for $|I| \leq 1$,

$$
\left|\left\langle\theta_{I}, \eta\right\rangle\right| \leq C \ell(I)^{r_{1}+d / 2}\left(1+\left|x_{I}\right|\right)^{-M} .
$$

Then

$$
\left|\sum_{I \in D} d_{I}\left\langle\theta_{I}, \eta\right\rangle\right| \leq \sum_{|I|>1}\left|d_{I}\right|\left|\left\langle\theta_{I}, \eta\right\rangle\right|+\sum_{|I| \leq 1}\left|d_{I}\right|\left|\left\langle\theta_{I}, \eta\right\rangle\right|=: \sigma_{1}+\sigma_{2} .
$$

Using the fact that $\left|d_{I}\right| \leq C|I|^{s / d+1 / 2-1 / p}$ for every $I \in D$, we get

$$
\begin{aligned}
\sigma_{1} & \leq C \sum_{|I|>1}|I|^{s / d+1 / 2-1 / p-(k+1) / d-1 / 2}\left(1+\left|x_{I}\right| / \ell(I)\right)^{-M} \\
& \leq C \sum_{m \geq 0} 2^{m(s-k-1-d / p)} \sum_{I \in D_{m}}\left(1+\left|x_{I}\right| / \ell(I)\right)^{-M} \\
& \leq C \sum_{m \geq 0} 2^{m(s-k-1-d / p)}<\infty
\end{aligned}
$$

because $k+1>s-d / p$.

To estimate $\sigma_{2}$ we will use the maximal operator $M_{t}$ defined by

$$
M_{t}(f)(x):=\left(\sup _{Q \ni x}|Q|^{-1} \int_{Q}|f(y)|^{t} d y\right)^{1 / t},
$$

where the sup is taken over all cubes $Q$ (containing $x$ ) with sides parallel to the axes, and $t$ is selected so that $0<t<\min \{1, p, q\}, M>d / t$, and $r_{1}>d / t-d-s$. Using Lemma 7.1 from Appendix II we infer that for every $x$ in the unit cube $I_{0}$,

$$
\begin{aligned}
\sigma_{2} & =C \sum_{|I| \leq 1}|I|^{r_{1} / d+1 / 2}\left|d_{I}\right|\left(1+\left|x_{I}\right|\right)^{-M} \\
& \leq C \sum_{m \geq 0} 2^{-m\left(r_{1}+d / 2\right)} \sum_{I \in D_{m}}\left|d_{I}\right|\left(1+\left|x_{I}\right|\right)^{-M} \\
& \leq C \sum_{m \geq 0} 2^{-m\left(r_{1}+d / 2-d / t\right)} M_{t}\left(\sum_{I \in D_{m}}\left|d_{I}\right| \chi_{I}\right)(x) .
\end{aligned}
$$

We let $\lambda_{I}:=|I|^{-s / d-1 / 2} \chi_{I}$. Since $r_{1}>d / t-d-s$, for $x \in I_{0}$ we have

$$
\begin{aligned}
\sigma_{2} & \leq C \sum_{m \geq 0} 2^{-\mu\left(r_{1}+s+d-d / t\right)} M_{t}\left(\sum_{I \in D_{m}}\left|d_{I}\right| \lambda_{I}\right)(x) \\
& \leq C \sup _{m \geq 0} M_{t}\left(\sum_{I \in D_{m}}\left|d_{I}\right| \lambda_{I}\right)(x) \leq C\left(\sum_{m \geq 0}\left(M_{t}\left(\sum_{I \in D_{m}}\left|d_{I}\right| \widetilde{\lambda}_{I}\right)(x)\right)^{q}\right)^{1 / q} .
\end{aligned}
$$


Taking the $L_{p}\left(I_{0}\right)$ norm and using the maximal inequality (7.1) we get

$$
\sigma_{2} \leq C\left\|\left(\sum_{m \geq 0}\left(M_{t}\left(\sum_{I \in D_{m}}\left|d_{I}\right| \widetilde{\lambda}_{I}\right)\right)^{q}\right)^{1 / q}\right\|_{L_{p}\left(I_{0}\right)} \leq C\|d\|_{\dot{f}_{p q}^{s}}<\infty .
$$

Thus, the series $f:=\sum_{I \in D} d_{I} \theta_{I}$ converges in $\mathcal{S}^{\prime} / \mathcal{P}_{k}$.

It remains to prove (4.8). If $c(I, J):=\left\langle\theta_{I}, \phi_{J}\right\rangle$, from the above we find

$$
s_{J}:=s_{J}(f):=\left\langle f, \phi_{J}\right\rangle=\sum_{I \in D} d_{I}\left\langle\theta_{I}, \phi_{J}\right\rangle=\sum_{I \in D} d_{I} c(I, J), \quad J \in D .
$$

In other words if $\varsigma:=\left(s_{J}\right)$ and $\mathbf{C}:=(c(I, J))_{I, J \in D}$, we have

$$
\varsigma=\mathbf{C}^{T} d \text {. }
$$

By Proposition 3.1, $\mathbf{C}^{T}$ is bounded on $\dot{f}_{p q}^{s}$. Therefore, using (2.17), we have

$$
\|f\|_{\dot{F}_{p q}^{s}} \approx\|\varsigma\|_{\dot{f}_{p q}^{s}}=\left\|\mathbf{C}^{T} d\right\|_{\dot{f}_{p q}^{s}} \leq C\|d\|_{\dot{f}_{p q}^{s}}
$$

Finally we note that once (4.8) has been established it follows that for $q \neq \infty$ the series $\sum_{I \in D} d_{I} \theta_{I}$ converges in the sense of $\dot{F}_{p q}^{s}$, since its tail $\sum_{|I| \geq N} d_{I} \theta_{I}$ converges strongly to 0 as $N \rightarrow \infty$.

LEMMA 4.2. Let $s \in \mathbb{R}, 0<p, q \leq \infty, \mathcal{J}:=d / \min \{1, p\}$ and $k:=$ $\max \{[s-d / p],-1\}$. If $\left(\theta_{I}\right)_{I \in D}$ satisfies $(3.5)-(3.6)$ for some $r_{1}, r_{2} \in \mathbb{N}$ with $r_{1}>\mathcal{J}-d-s, r_{2}>s$ and $M>\max \left\{\mathcal{J}, d+r_{1}, d+r_{2}\right\}$, then for every $d:=\left(d_{I}\right) \in \dot{b}_{p q}^{s}$ the series $\sum_{I \in D} d_{I} \theta_{I}$ converges in $\mathcal{S}^{\prime} / \mathcal{P}_{k}$ (and in $\dot{B}_{p q}^{s}$ for $p, q \neq \infty)$ and

$$
\left\|\sum_{I \in D} d_{I} \theta_{I}\right\|_{\dot{B}_{p q}^{s}} \leq C\|d\|_{\dot{b}_{p q}^{s}}
$$

Proof. The proof of (4.11) is identical to the one of (4.8) since under our assumptions the matrix $\mathbf{C}^{T}$ is bounded on $\dot{b}_{p q}^{s}$. Therefore, we need only establish that the series $\sum_{I \in D} d_{I} \theta_{I}$ converges in $\mathcal{S}^{\prime} / \mathcal{P}_{k}$. For this, we note that for every $\eta \in S_{k}$,

$$
\left|\sum_{I \in D} d_{I}\left\langle\theta_{I}, \eta\right\rangle\right| \leq \sum_{|I|>1}\left|d_{I}\right|\left|\left\langle\theta_{I}, \eta\right\rangle\right|+\sum_{|I| \leq 1}\left|d_{I}\right|\left|\left\langle\theta_{I}, \eta\right\rangle\right|=: \sigma_{1}+\sigma_{2} .
$$

Since $d \in \dot{b}_{p q}^{s}$, we have $\left|d_{I}\right| \leq C|I|^{s / d+1 / 2-1 / p}, I \in D$, and the proof of Lemma 4.1 shows immediately that $\sigma_{1}<\infty$.

For $\sigma_{2}$, we will consider two cases.

CASE I: $0<p \leq 1$. We have

$$
\begin{aligned}
\sigma_{2} & \leq \sum_{|I| \leq 1}|I|^{r_{1} / d+1 / 2}\left|d_{I}\right|\left(1+\left|x_{I}\right|\right)^{-M} \\
& \leq \sum_{m \geq 0} 2^{-m\left(r_{1}+s+d-d / p\right)} \sum_{I \in D_{m}}|I|^{-s / d-1 / 2+1 / p}\left|d_{I}\right|
\end{aligned}
$$




$$
\leq \sup _{m \geq 0}\left(\sum_{I \in D_{m}}\left(|I|^{-s / d-1 / 2+1 / p}\left|d_{I}\right|\right)^{p}\right)^{1 / p} \leq C\|d\|_{\dot{b}_{p q}^{s}}<\infty
$$

where we used $r_{1}>\mathcal{J}-d-s$ and $\sum\left|x_{j}\right| \leq\left(\sum\left|x_{j}\right|^{p}\right)^{1 / p}, 0<p \leq 1$.

CASE II: $p>1$. From Hölder's inequality and

$$
\sum_{I \in D_{m}}\left(1+\left|x_{I}\right|\right)^{-M} \leq C 2^{m d}, \quad m \geq 0, M>d
$$

we obtain

$$
\begin{aligned}
\sigma_{2} & =\sum_{|I| \leq 1}|I|^{r_{1} / d+1 / 2}\left|d_{I}\right|\left(1+\left|x_{I}\right|\right)^{-M} \\
& \leq \sum_{m \geq 0} 2^{-m\left(r_{1}+d / 2\right)}\left(\sum_{I \in D_{m}}\left|d_{I}\right|^{p}\right)^{1 / p}\left(\sum_{I \in D_{m}}\left(1+\left|x_{I}\right|\right)^{-M p^{\prime}}\right)^{1 / p^{\prime}} \\
& \leq C \sum_{m \geq 0} 2^{-m\left(r_{1}+d / 2-d / p^{\prime}\right)}\left(\sum_{I \in D_{m}}\left|d_{I}\right|^{p}\right)^{1 / p} \\
& =C \sum_{m \geq 0} 2^{-m\left(r_{1}+s\right)}\left(\sum_{I \in D_{m}}\left(|I|^{-s / d-1 / 2+1 / p}\left|d_{I}\right|\right)^{p}\right)^{1 / p} \leq C\|d\|_{\dot{b}_{p q}^{s}}<\infty,
\end{aligned}
$$

where $1 / p^{\prime}:=1-1 / p$. This concludes the proof of the lemma.

REMARK 4.1. Assuming that $\left(d_{I}\right)_{I \in D}$ and $\left(\theta_{I}\right)_{I \in D}$ satisfy the assumptions of Lemma 4.1 (or Lemma 4.2), it is obvious from its proof that the series $\sum_{I \in D} d_{I}\left\langle\theta_{I}, \eta\right\rangle$ converges not only for $\eta \in \mathcal{S}_{k}, k:=\max \{[s-d / p],-1\}$, but for any function $\eta$ which has $k$ zero moments and satisfies

$$
\left|\eta^{(\alpha)}(x)\right| \leq C(1+|x|)^{-M}, \quad|\alpha| \leq r_{1}
$$

(with $r_{1}, M$ as in Lemma 4.1 or Lemma 4.2 ). Therefore if $f:=\sum_{I \in D} d_{I} \theta_{I}$ we may define

$$
\langle f, \eta\rangle:=\sum_{I \in D} d_{I}\left\langle\theta_{I}, \eta\right\rangle .
$$

REMARK 4.2. When $s-d / p \in \mathbb{N}$ and $0<p, q \leq 1$ it can be shown that the series $\sum_{I \in D} d_{I} \theta_{I}$ in Lemma 4.1 converges in $\mathcal{S}^{\prime} / \mathcal{P}_{k}$ with $k=s-d / p-1$ and as a consequence the same holds for the series in Theorem 4.1 and the wavelet series in Proposition 4.1. A similar result holds, in the case of Besov spaces, regarding the convergence of the corresponding series in Lemma 4.2, Theorem 4.2 and the wavelet series in Proposition 4.2, under the assumptions that $s-d / p \in \mathbb{N}$ and $0<q \leq 1$.

For every $e \in E$ we recall from $\S 3$ the matrices

$$
\begin{array}{ll}
\widetilde{\mathbf{A}}_{e}:=\left(\widetilde{a}_{e}(I, J)\right)_{I, J \in D}, & \text { where } \widetilde{a}_{e}(I, J)=\left\langle\phi_{I}, \widetilde{\theta}_{J}^{e}\right\rangle, \\
\mathbf{A}_{e}:=\left(a_{e}(I, J)\right)_{I, J \in D}, & \text { where } a_{e}(I, J)=\left\langle\theta_{I}^{e}, \phi_{J}\right\rangle .
\end{array}
$$


Corollary 4.1. Let $\theta_{I}^{e}, \widetilde{\theta}_{I}^{e}, I \in D, e \in E$, satisfy the assumptions of Theorem 4.1 (or Theorem 4.2). Then

$$
\phi_{I}=\sum_{e \in E} \sum_{J \in D}\left\langle\phi_{I}, \widetilde{\theta}_{J}^{e}\right\rangle \theta_{J}^{e}, \quad I \in D,
$$

where the convergence is in $\mathcal{S}^{\prime}$, as well as in $\dot{F}_{p q}^{s}\left(\right.$ resp. $\left.\dot{B}_{p q}^{s}\right)$ for $p, q \neq \infty$.

Proof. Since $B$ is a decomposition system for $L_{2}\left(\mathbb{R}^{d}\right)$, (4.14) holds immediately in the $L_{2}$ sense and consequently in the distributional sense. To show that the convergence of the series also holds in the sense of $\dot{F}$ or $\dot{B}$ spaces, it is sufficient by Lemmata 4.1, 4.2 to show that for each $I \in D$ and $e \in E$ the sequence $\left(\widetilde{a}_{e}(I, J)\right)_{J}$ is in $\dot{f}_{p q}^{s}$ (or $\left.\dot{b}_{p q}^{s}\right)$. For this we define the sequence $\delta^{I}:=\left(\delta_{I, J}\right)$ by

$$
\delta_{I, J}:= \begin{cases}1, & I=J \\ 0, & I \neq J\end{cases}
$$

Then $\left(\widetilde{a}_{e}(I, J)\right)_{J}=\widetilde{\mathbf{A}}_{e}^{T} \delta^{I}$. By Proposition 3.1 (or 3.2), $\widetilde{\mathbf{A}}_{e}^{T}$ is bounded on $\dot{f}_{p q}^{s}\left(\right.$ or $\left.\dot{b}_{p q}^{s}\right)$ and consequently $\left(\widetilde{a}_{e}(I, J)\right)_{J} \in \dot{f}_{p q}^{s}$ (resp. $\left.\in \dot{b}_{p q}^{s}\right)$.

Proof of Theorem 4.1. We are going to establish that for every $f \in \dot{F}_{p q}^{s}$,

$$
\begin{gathered}
f=\sum_{e \in E} \sum_{J \in D}\left\langle f, \widetilde{\theta}_{J}^{e}\right\rangle \theta_{J}^{e}, \\
\|f\|_{\dot{F}_{p q}^{s}} \approx \sum_{e \in E}\left\|\left(\widetilde{a}_{I}^{e}(f)\right)_{I}\right\|_{\dot{f}_{p q}^{s}} .
\end{gathered}
$$

From (2.16) and Corollary 4.1 we get

$$
\begin{aligned}
f & =\sum_{I \in D} s_{I}(f) \phi_{I}=\sum_{I \in D} \sum_{e \in E} \sum_{J \in D} s_{I}(f) \widetilde{a}_{e}(I, J) \theta_{J}^{e} \\
& =\sum_{e \in E} \sum_{J \in D} \sum_{I \in D} s_{I}(f) \widetilde{a}_{e}(I, J) \theta_{J}^{e} \\
& =\sum_{e \in E} \sum_{J \in D} \sum_{I \in D}\left\langle f, \phi_{I}\right\rangle\left\langle\phi_{I}, \widetilde{\theta}_{J}^{e}\right\rangle \theta_{J}^{e}=\sum_{e \in E} \sum_{J \in D}\left\langle f, \widetilde{\theta}_{J}^{e}\right\rangle \theta_{J}^{e},
\end{aligned}
$$

where all identities above are considered in the distributional sense. To justify the third equality, we note that the assumptions of the theorem guarantee that for every $e \in E$ the matrix $\widetilde{\mathbf{A}}_{e}^{T}$ is bounded on $\dot{f}_{p q}^{s}$. Since $\varsigma:=\left(s_{I}(f)\right)_{I} \in \dot{f}_{p q}^{s}$, the sequence $\left(d_{J}^{e}\right):=\left(\sum_{I \in D}\left|\widetilde{a}_{e}(I, J)\right|\left|s_{I}(f)\right|\right)_{J}$ belongs in $\dot{f}_{p q}^{s}$. Finally, for every $\eta \in \mathcal{S}_{k}$ with $k:=\max \{[s-d / p],-1\}$, as in the proof of Lemma 4.1 we have

$$
\sum_{J \in D}\left|d_{J}^{e}\right|\left|\left\langle\theta_{J}^{e}, \eta\right\rangle\right|<\infty
$$

which allows us to interchange the order of the summations. 
Next, we will prove the norm equivalence (4.16). From (4.17) we get

$$
s_{I}(f)=\sum_{e \in E} \sum_{J \in D}\left\langle f, \widetilde{\theta}_{J}^{e}\right\rangle\left\langle\theta_{J}^{e}, \phi_{I}\right\rangle=\sum_{e \in E} \sum_{J \in D} a_{e}(J, I) \widetilde{a}_{J}^{e}(f), \quad I \in D .
$$

Setting $\widetilde{a}_{e}:=\left(\widetilde{a}_{I}^{e}(f)\right)_{I}$ we can rewrite $(4.18)$ in the form

$$
\varsigma=\sum_{e \in E} \mathbf{A}_{e}^{T} \widetilde{a}_{e} .
$$

Similarly

$$
\widetilde{a}_{I}^{e}(f)=\sum_{J \in D} \widetilde{a}_{e}(J, I) s_{J}(f), \quad I \in D, e \in E
$$

i.e.,

$$
\widetilde{a}_{e}=\widetilde{\mathbf{A}}_{e}^{T} \varsigma, \quad e \in E .
$$

Since by Proposition 3.1, $\widetilde{\mathbf{A}}_{e}^{T}, \mathbf{A}_{e}^{T}, e \in E$, are bounded on $\dot{f}_{p q}^{s}$ it follows that

$$
\begin{aligned}
\|\varsigma\|_{\dot{f}_{p q}^{s}} & =\left\|\sum_{e \in E} \mathbf{A}_{e}^{T} \widetilde{a}_{e}\right\|_{\dot{f}_{p q}^{s}} \leq C \sum_{e \in E}\left\|\mathbf{A}_{e}^{T} \widetilde{a}_{e}\right\|_{\dot{f}_{p q}^{s}} \leq C \sum_{e \in E}\left\|\widetilde{a}_{e}\right\|_{\dot{f}_{p q}^{s}} \\
& =C \sum_{e \in E}\left\|\widetilde{\mathbf{A}}_{e}^{T} s\right\|_{\dot{f}_{p q}^{s}} \leq C\|\varsigma\|_{\dot{f}_{p q}^{s}} .
\end{aligned}
$$

This concludes the proof of the theorem.

Proof of Theorem 4.2. The proof is identical to that of Theorem 4.1, one only needs to change $\dot{f}_{p q}^{s}$ to $\dot{b}_{p q}^{s}$ and use Proposition 3.2 and Lemma 4.2 instead of Proposition 3.1 and Lemma 4.1.

5. Inhomogeneous spaces. As we already stated in the introduction, the results of the previous section hold for the inhomogeneous TriebelLizorkin and Besov spaces as well. For notational reasons we present them here only within the framework of the biorthogonal wavelet bases $W_{0}$ described in the introduction. Since the proofs of these results are identical to the ones already given, we restrict ourselves to highlighting the only difference in the assumptions on the wavelet sets $W$ and $W_{0}$, namely the fact that the wavelets $\psi^{\mathbf{0}}, \widetilde{\psi}^{\mathbf{0}}$ do not satisfy the zero moments condition $\mathbf{B 2}$ '.

For the definition of these spaces we start with the family of functions $\phi_{\nu}, \nu \in \mathbb{Z}$, which satisfies (2.1)(i), (ii) and we define a function $\Phi \in \mathcal{S}$ such that $\operatorname{supp} \widehat{\Phi} \subset\{\xi:|\xi| \leq 2\}$ and

$$
|\widehat{\Phi}(\xi)|^{2}+\sum_{\nu \geq 1}\left|\widehat{\phi}_{\nu}(\xi)\right|^{2}=1 .
$$

For $s \in \mathbb{R}, 0<p<\infty, 0<q \leq \infty$, the inhomogeneous Triebel-Lizorkin space $F_{p q}^{s}$ is defined to be the set of all $f \in \mathcal{S}^{\prime}$ such that 


$$
\|f\|_{F_{p q}^{s}}:=\|f * \Phi\|_{L_{p}}+\left\|\left(\sum_{\nu \geq 1}\left(2^{\nu s}\left|\phi_{\nu} * f\right|\right)^{q}\right)^{1 / q}\right\|_{L_{p}}<\infty
$$

(with the usual modification for $q=\infty$ ).

Similarly for $s \in \mathbb{R}, 0<p, q \leq \infty$, the inhomogeneous Besov space $B_{p q}^{s}$ is defined to be the set of all $f \in \mathcal{S}^{\prime}$ such that

$$
\|f\|_{B_{p q}^{s}}:=\|f * \Phi\|_{L_{p}}+\left(\sum_{\nu \geq 1}\left(2^{\nu s}\left\|\phi_{\nu} * f\right\|_{L_{p}}\right)^{q}\right)^{1 / q}<\infty .
$$

From (5.1) one can prove that for every $f \in \mathcal{S}^{\prime}$,

$$
f=\widetilde{\Phi} * \Phi * f+\sum_{\nu \geq 1} \widetilde{\phi}_{\nu} * \phi_{\nu} * f
$$

in the sense of $\mathcal{S}^{\prime}(\widetilde{\Phi}(x):=\overline{\Phi(-x)})$. Also, similarly to (2.16),

$$
f=\sum_{|I|=1}\left\langle f, \Phi_{I}\right\rangle \Phi_{I}+\sum_{|I|<1}\left\langle f, \phi_{I}\right\rangle \phi_{I} .
$$

Let us now define the sequence $\varsigma:=\left(s_{I}\right)_{I \in D_{+}}$with

$$
s_{I}:=s_{I}(f):= \begin{cases}\left\langle f, \Phi_{I}\right\rangle, & |I|=1, \\ \left\langle f, \phi_{I}\right\rangle, & |I|<1 .\end{cases}
$$

It turns out that $f \in F_{p q}^{s}$ if and only if $\varsigma \in f_{p q}^{s}$, where for any sequence $h:=\left(h_{I}\right)_{I \in D_{+}}$and $s \in \mathbb{R}, 0<p<\infty, 0<q \leq \infty$,

$$
\|h\|_{f_{p q}^{s}}:=\left\|\left(\sum_{I \in D_{+}}\left(|I|^{-s / d}\left|h_{I}\right| \widetilde{\chi}_{I}\right)^{q}\right)^{1 / q}\right\|_{L_{p}}<\infty .
$$

In particular it was shown in [FJ2] that

$$
\|f\|_{F_{p q}^{s}} \approx\|\varsigma\|_{f_{p q}^{s}} .
$$

Similarly, membership in $B_{p q}^{s}, s \in \mathbb{R}, 0<p, q \leq \infty$, can be determined by the size of the sequence $\varsigma$ measured in the (quasi)norm $\|\cdot\|_{b_{p q}^{s}}$ defined by

$$
\|h\|_{b_{p q}^{s}}:=\left(\sum_{m \geq 0}\left(\sum_{I \in D_{m}}\left(|I|^{-s / d+1 / p-1 / 2}\left|h_{I}\right|\right)^{p}\right)^{q / p}\right)^{1 / q}<\infty .
$$

In other words (see [FJ1]),

$$
\|f\|_{B_{p q}^{s}} \approx\|\varsigma\|_{b_{p q}^{s}} .
$$

We are now ready to state the main results of the section:

Proposition 5.1. Let $s \in \mathbb{R}, 0<p<\infty, 0<q \leq \infty, \mathcal{J}:=d / \min \{1, p, q\}$. Let also $\Psi, \widetilde{\Psi}$ be dual wavelet sets for $L_{2}\left(\mathbb{R}^{d}\right)$ which satisfy (1.13) and (1.14) for some $r_{\Psi}, r_{\widetilde{\Psi}} \in \mathbb{N}_{+}$with $r_{\Psi}>s, r_{\widetilde{\Psi}}>\mathcal{J}-d-s$ and $M_{\Psi}, M_{\widetilde{\Psi}}>\max \{\mathcal{J}$, 
$\left.d+r_{\Psi}, d+r_{\widetilde{\Psi}}\right\}$. Then, for every $f \in F_{p q}^{s}$, there exist unique coefficients

$$
\widetilde{c}_{I}^{e}(f):=\left\langle f, \widetilde{\psi}_{I}^{e}\right\rangle, \quad e \in E_{0}, I \in D_{+},
$$

such that

$$
f=\sum_{I \in D_{0}} \widetilde{c}_{I}^{\mathbf{0}}(f) \psi_{I}^{\mathbf{0}}+\sum_{e \in E} \sum_{I \in D_{+}} \widetilde{c}_{I}^{e}(f) \psi_{I}^{e}
$$

in the sense of $\mathcal{S}^{\prime}$ (and in $F_{p q}^{s}$ for $q \neq \infty$ ). Moreover,

$$
\|f\|_{F_{p q}^{s}} \approx\left(\sum_{I \in D_{0}}\left|\widetilde{c}_{I}^{0}(f)\right|^{p}\right)^{1 / p}+\sum_{e \in E}\left\|\left(\widetilde{c}_{I}^{e}(f)\right)_{I}\right\|_{f_{p q}^{s}} .
$$

Proposition 5.2. Let $s \in \mathbb{R}, 0<p, q \leq \infty, \mathcal{J}:=d / \min \{1, p\}$. Let also $\Psi, \widetilde{\Psi}$ be dual wavelet sets for $L_{2}\left(\mathbb{R}^{d}\right)$ which satisfy (1.13) and (1.14) for some $r_{\Psi}, r_{\widetilde{\Psi}} \in \mathbb{N}_{+}$with $r_{\Psi}>s, r_{\widetilde{\Psi}}>\mathcal{J}-d-s$ and $M_{\Psi}, M_{\widetilde{\Psi}}>\max \left\{\mathcal{J}, d+r_{\Psi}\right.$, $\left.d+r_{\widetilde{\Psi}}\right\}$. Then, for every $f \in B_{p q}^{s}$, there exist unique coefficients $\widetilde{c}_{I}^{e}(f)$, $e \in E_{0}, I \in D_{+}$, given by (5.7) such that

$$
f=\sum_{I \in D_{0}} \widetilde{c}_{I}^{\mathbf{0}}(f) \psi_{I}^{\mathbf{0}}+\sum_{e \in E} \sum_{I \in D_{+}} \widetilde{c}_{I}^{e}(f) \psi_{I}^{e}
$$

in the sense of $\mathcal{S}^{\prime}$ (and in $B_{p q}^{s}$ for $p, q \neq \infty$ ). Moreover,

$$
\|f\|_{B_{p q}^{s}} \approx\left(\sum_{I \in D_{0}}\left|\widetilde{c}_{I}^{0}(f)\right|^{p}\right)^{1 / p}+\sum_{e \in E}\left\|\left(\widetilde{c}_{I}^{e}(f)\right)_{I}\right\|_{b_{p q}^{s}} .
$$

Since the family

$$
W_{0}=\left\{\psi_{I}^{\mathbf{0}}, \widetilde{\psi}_{I}^{\mathbf{0}}: I \in D_{0}\right\} \cup\left\{\psi_{I}^{e}, \widetilde{\psi}_{I}^{e}: I \in D_{+}, e \in E\right\},
$$

forms an unconditional basis for $L_{2}\left(\mathbb{R}^{d}\right)$, for every $I \in D_{0}$ we have

$$
\Phi_{I}=\sum_{J \in D_{0}} \widetilde{a}_{\mathbf{0}}(I, J) \psi_{J}^{\mathbf{0}}+\sum_{e \in E} \sum_{J \in D_{+}} \widetilde{a}_{e}(I, J) \psi_{J}^{e},
$$

and similarly for $|I|<1$,

$$
\phi_{I}=\sum_{J \in D_{0}} \widetilde{a}_{\mathbf{0}}(I, J) \psi_{J}^{\mathbf{0}}+\sum_{e \in E} \sum_{J \in D_{+}} \widetilde{a}_{e}(I, J) \psi_{J}^{e},
$$

where for $e \in E$,

$$
\widetilde{a}_{e}(I, J):= \begin{cases}\left\langle\Phi_{I}, \widetilde{\psi}_{J}^{e}\right\rangle, & |I|=1,|J| \leq 1, \\ \left\langle\phi_{I}, \widetilde{\psi}_{J}^{e}\right\rangle, & |I|<1,|J| \leq 1,\end{cases}
$$

while

$$
\widetilde{a}_{\mathbf{0}}(I, J):= \begin{cases}\left\langle\Phi_{I}, \widetilde{\psi}_{J}^{\mathbf{0}}\right\rangle, & |I|=1,|J|=1, \\ \left\langle\phi_{I}, \widetilde{\psi}_{J}^{\mathbf{0}}\right\rangle, & |I|<1,|J|=1, \\ 0, & |I| \leq 1,|J|<1 .\end{cases}
$$


Similarly, using (5.4), for every $e \in E_{0}, I \in D_{+}$we have

$$
\psi_{I}^{e}=\sum_{|J|=1} a_{e}(I, J) \Phi_{J}+\sum_{|J|<1} a_{e}(I, J) \phi_{J},
$$

where for $e \in E$,

$$
a_{e}(I, J):= \begin{cases}\left\langle\psi_{I}^{e}, \Phi_{J}\right\rangle, & |I| \leq 1,|J|=1 \\ \left\langle\psi_{I}^{e}, \phi_{J}\right\rangle, & |I| \leq 1,|J|<1\end{cases}
$$

and

$$
a_{\mathbf{0}}(I, J):= \begin{cases}\left\langle\psi_{I}^{\mathbf{0}}, \Phi_{J}\right\rangle, & |I|=1,|J|=1 \\ \left\langle\psi_{I}^{\mathbf{0}}, \phi_{J}\right\rangle, & |I|=1,|J|<1 \\ 0, & |I|<1,|J| \leq 1\end{cases}
$$

Similarly to Propositions 4.1, 4.2, we note that the proofs of both propositions above are based on the fact that the transposes of the transformation matrices

$$
\widetilde{\mathbf{A}}_{e}:=\left(\widetilde{a}_{e}(I, J)\right)_{I, J \in D_{+}}, \quad \mathbf{A}_{e}:=\left(a_{e}(I, J)\right)_{I, J \in D_{+}}, \quad e \in E_{0},
$$

are bounded on $f_{p q}^{s}$ and $b_{p q}^{s}$, for the range of the indices $s, p, q$ given above. To prove this, one has essentially to establish the inhomogeneous versions of Propositions 3.1, 3.2. Going a step further, we note that in order to estimate the size of the entries of the above matrices one uses Lemma 3.1 and the only point of possible concern is that $\psi^{\mathbf{0}}, \widetilde{\psi}^{\mathbf{0}}$ and $\Phi$ do not have any vanishing moments. However, we recall (see Remark 3.2) that the size of $\left|\left\langle\theta_{I}, \eta_{J}\right\rangle\right|$ when $|J| \leq|I|$ depends on the zero moments of $\eta_{J}$ and the smoothness of $\theta_{I}$. Taking a closer look at the above matrices we see that, indeed, all three functions $\psi^{\mathbf{0}}, \widetilde{\psi}^{\mathbf{0}}$ and $\Phi$ are indexed only by $D_{0}$ and thus will play the role of $\theta_{I}$, except for $\left\langle\psi_{I}^{\mathbf{0}}, \Phi_{J}\right\rangle$ or $\left\langle\Phi_{I}, \widetilde{\psi}_{J}^{\mathbf{0}}\right\rangle$ with $I, J \in D_{0}$, in which case one has to use Remark 3.1.

6. Appendix I: Calderón's formula. Our goal is to shed some light on the reproduction formula (2.4). For $\eta \in \mathcal{S}$ it is not hard to see that

$$
\sum_{\nu \in \mathbb{Z}} \widetilde{\phi}_{\nu} * \phi_{\nu} * \eta(x)=\eta(x), \quad x \in \mathbb{R}^{d} .
$$

Indeed, since $\eta, \widehat{\eta} \in L_{1}\left(\mathbb{R}^{d}\right)$, by Fourier inversion, for every $x \in \mathbb{R}^{d}$ and $N, K \geq 0$,

$$
\begin{aligned}
& \lim _{N, K \rightarrow \infty}\left|\sum_{-N \leq \nu \leq K} \widetilde{\phi}_{\nu} * \phi_{\nu} * \eta(x)-\eta(x)\right| \\
& \leq \lim _{N, K \rightarrow \infty} \int_{\mathbb{R}^{d}}\left(1-\sum_{-N \leq \nu \leq K}\left|\widehat{\phi}_{\nu}(\xi)\right|^{2}\right)|\widehat{\eta}(\xi)| d \xi=0
\end{aligned}
$$

where the last equality holds by Lebesgue's convergence theorem. 
Lemma 6.1. Suppose that $\eta \in \mathcal{S}_{k}$ with $k \geq-1\left(\mathcal{S}_{-1}:=\mathcal{S}\right)$. Then there exists a constant $C$ such that

$$
\sum_{\nu \in \mathbb{Z}}\left|\widetilde{\phi}_{\nu} * \phi_{\nu} * \eta(x)\right| \leq C(1+|x|)^{-(d+k+1)}, \quad x \in \mathbb{R}^{d} .
$$

Proof. Use the fact that if $\nu>0$ then

$$
\left|\widetilde{\phi}_{\nu} * \phi_{\nu} * \eta(x)\right| \leq C 2^{-\nu}(1+|x|)^{-M}, \quad x \in \mathbb{R}^{d},
$$

while for $\nu \leq 0$,

$$
\left|\widetilde{\phi}_{\nu} * \phi_{\nu} * \eta(x)\right| \leq C 2^{\nu(d+k+1)}\left(1+2^{\nu}|x|\right)^{-M}, \quad x \in \mathbb{R}^{d},
$$

for any $M>0$. We leave the details to the reader and we refer to [FJW, Appendix III] for the proof of the continuous version of this lemma.

Proposition 6.1. Let $k \geq-1$ and $f \in \mathcal{S}^{\prime}$ be such that

Then

$$
\int_{\mathbb{R}^{d}}|f(x)|(1+|x|)^{-(d+k+1)} d x<\infty .
$$

$$
\sum_{\nu \in \mathbb{Z}} \widetilde{\phi}_{\nu} * \phi_{\nu} * f=f
$$

in the sense of $\left(\mathcal{S}_{k}\right)^{\prime}$.

Proof. Let $\eta \in \mathcal{S}_{k}$. Since $\left\langle\widetilde{\phi}_{\nu} * \phi_{\nu} * f, \eta\right\rangle=\left\langle f, \widetilde{\phi}_{\nu} * \phi_{\nu} * \eta\right\rangle$ we need to establish that

$$
\lim _{N, K \rightarrow \infty} \int_{\mathbb{R}^{d}} f(x) \overline{\eta(x)-\sum_{-N \leq \nu \leq K} \widetilde{\phi}_{\nu} * \phi_{\nu} * \eta(x)} d x=0 .
$$

From (6.1),

$$
\begin{aligned}
& \left|\int_{\mathbb{R}^{d}} f(x) \overline{\eta(x)-\sum_{-N \leq \nu \leq K} \widetilde{\phi}_{\nu} * \phi_{\nu} * \eta(x)} d x\right| \\
& \leq \int_{\mathbb{R}^{d}}|f(x)|\left(\sum_{\nu<-N}+\sum_{\nu>K}\right)\left|\widetilde{\phi}_{\nu} * \phi_{\nu} * \eta(x)\right| d x \\
& =\int_{\mathbb{R}^{d}} \frac{|f(x)|}{(1+|x|)^{d+k+1}} g_{N, K}(x) d x,
\end{aligned}
$$

where $g_{N, K}(x):=(1+|x|)^{d+k+1}\left(\sum_{\nu<-N}+\sum_{\nu>K}\right)\left|\widetilde{\phi}_{\nu} * \phi_{\nu} * \eta(x)\right|$. Moreover, from $(6.2)$,

$$
\lim _{N, K \rightarrow \infty} g_{N, K}(x)=0 .
$$

Applying Lebesgue's convergence theorem yields the result.

For each $\eta \in \mathcal{S}$ and $k, N \in \mathbb{N}$ we define

$$
p_{k, N}(\eta):=\sup _{|\alpha| \leq N} \sup _{x \in \mathbb{R}^{d}}(1+|x|)^{k}\left|\eta^{(\alpha)}(x)\right| .
$$

A useful consequence of Proposition 6.1 is the following: 
Proposition 6.2. Let $k, N \in \mathbb{N}$ and suppose that $f \in \mathcal{S}^{\prime}$ is such that (6.6)

$$
|\langle f, \eta\rangle| \leq C p_{k, N}(\eta), \quad \eta \in \mathcal{S} .
$$

Then

$$
\sum_{\nu \in \mathbb{Z}} \widetilde{\phi}_{\nu} * \phi_{\nu} * f=f
$$

in the sense of $\left(\mathcal{S}_{k}\right)^{\prime}$.

Proof. Let

$$
\widehat{\Phi}:=1-\sum_{\nu \geq 0}\left|\widehat{\phi}_{\nu}\right|^{2}
$$

Since $\sum_{\nu \in \mathbb{N}}\left|\widehat{\phi}_{\nu}(\xi)\right|=1, \xi \neq 0$,

$$
f=f * \Phi+\sum_{\nu \geq 0} \widetilde{\phi}_{\nu} * \phi_{\nu} * f=: f_{1}+f_{2} .
$$

From our discussion in $\S 2$ we know that

$$
\sum_{\nu \in \mathbb{Z}} \widetilde{\phi}_{\nu} * \phi_{\nu} * f_{2}=\sum_{\nu \geq-1} \widetilde{\phi}_{\nu} * \phi_{\nu} * f_{2}=f_{2} \quad \text { in } \mathcal{S}^{\prime} .
$$

Thus, it is sufficient to show that

$$
\sum_{\nu \in \mathbb{Z}} \widetilde{\phi}_{\nu} * \phi_{\nu} * f_{1}=f_{1} \quad \text { in }\left(\mathcal{S}_{k}\right)^{\prime}
$$

However (6.6) implies that $f_{1}$ is of polynomial growth and in particular $\left|f_{1}(x)\right| \leq C|x|^{k}$ (see [R, p. 179]). The statement now follows immediately from Proposition 6.1.

For the next lemma it is useful to observe that an equivalent definition for $\mathcal{S}_{k}, k \in \mathbb{N}$, is given by

$$
\mathcal{S}_{k}:=\left\{\eta \in \mathcal{S}: \widehat{\eta}^{(\alpha)}(0)=0,|\alpha| \leq k\right\} .
$$

(This follows from the identity $\int x^{\alpha} \eta(x) d x=\widehat{x^{\alpha} \eta}(0)=(-i)^{-|\alpha|} \widehat{\eta}^{(\alpha)}(0)$.)

Lemma 6.2. Let $f \in \mathcal{S}^{\prime}$ and $k \geq-1$. Then the following are equivalent:

(i) $\sum_{\nu \in \mathbb{Z}} \widetilde{\phi}_{\nu} * \phi_{\nu} * f=g$ in $\left(\mathcal{S}_{k}\right)^{\prime}$.

(ii) There exist polynomials $P_{N}, P \in \mathcal{P}_{k}$ such that

$$
\lim _{N \rightarrow \infty} \sum_{\nu=-N}^{\infty} \widetilde{\phi}_{\nu} * \phi_{\nu} * f+P_{N}=g+P \quad \text { in } \mathcal{S}^{\prime} .
$$

(iii) For every $\alpha \in \mathbb{N}^{d}$ with $|\alpha|>k$, the series $\sum_{\nu<0}\left(\widetilde{\phi}_{\nu} * \phi_{\nu} * f\right)^{(\alpha)}$ converges in $\mathcal{S}^{\prime}$.

Proof. Since for $k=-1$ the result is trivial we assume that $k \in \mathbb{N}$. 
(i) $\Rightarrow$ (ii). Let $\theta \in \mathcal{S}$ be such that $\widehat{\theta}=1$ for $|\xi| \leq 2$ and $\widehat{\theta}=0$ for $|\xi|>4$. For $\nu>0$ we set $P_{\nu}=0$ while for $\nu \leq 0$ we define

(6.7) $P_{\nu}(x)=\sum_{|\alpha| \leq k} c_{\alpha, \nu} x^{\alpha}$, where $c_{\alpha, \nu}:=-\frac{(-i)^{|\alpha|}}{\alpha !}\left\langle\left|\widehat{\phi}_{\nu}(\xi)\right|^{2} \widehat{f}(\xi), \xi^{\alpha} \widehat{\theta}(\xi)\right\rangle$.

Then for every $\eta \in \mathcal{S}$ we have

$$
\begin{aligned}
\lim _{N \rightarrow \infty} \sum_{\nu=-N}^{\infty}\left\langle\widetilde{\phi}_{\nu} * \phi_{\nu} * f+P_{\nu}, \eta\right\rangle & \\
& =\sum_{\nu>0}\left\langle\widetilde{\phi}_{\nu} * \phi_{\nu} * f, \eta\right\rangle+\lim _{N \rightarrow \infty} \sum_{\nu=-N}^{0}\left\langle\widetilde{\phi}_{\nu} * \phi_{\nu} * f+P_{\nu}, \eta\right\rangle .
\end{aligned}
$$

There is no difficulty to prove that the first sum converges absolutely (see $\S 2)$. As far as the second sum is concerned, from the identity $\widehat{f}^{(\alpha)}(\cdot)=$ $\left((-i x)^{\alpha} f\right)^{\wedge}(\cdot)$ we get

$$
\left\langle P_{\nu}, \eta\right\rangle=\sum_{|\alpha| \leq k} c_{\alpha, \nu}(-i)^{-|\alpha|} \overline{\widehat{\eta}^{(\alpha)}(0)}
$$

It follows that

$$
\begin{aligned}
\lim _{N \rightarrow \infty} \sum_{\nu=-N}^{0} & \left\langle\widetilde{\phi}_{\nu} * \phi_{\nu} * f+P_{\nu}, \eta\right\rangle \\
& =\lim _{N \rightarrow \infty} \sum_{\nu=-N}^{0}\left(\left\langle\left|\widehat{\phi}_{\nu}\right|^{2} \widehat{f}, \widehat{\eta}\right\rangle+\left\langle P_{\nu}, \eta\right\rangle\right) \\
& =\lim _{N \rightarrow \infty} \sum_{\nu=-N}^{0}\left(\left\langle\left|\widehat{\phi}_{\nu}\right|^{2} \widehat{f}, \widehat{\theta} \widehat{\eta}\right\rangle+\sum_{|\alpha| \leq k} c_{\alpha, \nu}(-i)^{-|\alpha|} \overline{\widehat{\eta}^{(\alpha)}(0)}\right) \\
& =\lim _{N \rightarrow \infty} \sum_{\nu=-N}^{0}\left\langle\left|\widehat{\phi}_{\nu}(\xi)\right|^{2} \widehat{f}(\xi), \widehat{\theta}(\xi)\left(\widehat{\eta}(\xi)-\sum_{|\alpha| \leq k} \frac{\xi^{\alpha}}{\alpha !} \widehat{\eta}^{(\alpha)}(0)\right)\right\rangle \\
& =\lim _{N \rightarrow \infty} \sum_{\nu=-N}^{0}\left\langle\left|\widehat{\phi}_{\nu}(\xi)\right|^{2} \widehat{f}(\xi), \widehat{\omega}(\xi)\right\rangle=\sum_{\nu \leq 0}\left\langle\widetilde{\phi}_{\nu} * \phi_{\nu} * f, \omega\right\rangle,
\end{aligned}
$$

where $\widehat{\omega}(\xi)=\widehat{\theta}(\xi)\left(\widehat{\eta}(\xi)-\sum_{|\alpha| \leq k}\left(\xi^{\alpha} / \alpha !\right) \widehat{\eta}^{(\alpha)}(0)\right)$. Since $\omega \in \mathcal{S}_{k}$ we immediately see that the last sum above is finite. Thus

$$
h:=\lim _{N \rightarrow \infty}\left(\sum_{\nu=-N}^{\infty} \widetilde{\phi}_{\nu} * \phi_{\nu} * f+P_{N}\right)
$$

where $P_{N}=\sum_{\nu=-N}^{0} P_{\nu}$, defines a distribution in $\mathcal{S}^{\prime}$. We claim that $h-g$ 
$\in \mathcal{P}_{k}$. To see this we note that if $|\alpha|>k$ and $\eta \in \mathcal{S}$ then $\eta^{(\alpha)} \in \mathcal{S}_{k}$. Also $\operatorname{supp}(\widehat{h}-\widehat{g})=\{0\}$, i.e., $h-g$ is a polynomial which in addition vanishes on $\mathcal{S}_{k}$. Finally, since

$$
\left\langle(h-g)^{(\alpha)}, \eta\right\rangle=(-1)^{|\alpha|}\left\langle h-f, \eta^{(\alpha)}\right\rangle=0
$$

there exists $P \in \mathcal{P}_{k}$ such that $h=g+P$.

(ii) $\Rightarrow$ (i). This direction is trivial.

(i) $\Rightarrow$ (iii). Let $\eta \in \mathcal{S}$ and $|\alpha|>k$. Since $\eta^{(\alpha)} \in \mathcal{S}_{k}$,

$$
\begin{aligned}
\sum_{\nu \in \mathbb{Z}}\left\langle\left(\widetilde{\phi}_{\nu} * \phi_{\nu} * f\right)^{(\alpha)}, \eta\right\rangle & =(-1)^{|\alpha|} \sum_{\nu \in \mathbb{Z}}\left\langle\widetilde{\phi}_{\nu} * \phi_{\nu} * f, \eta^{(\alpha)}\right\rangle \\
& =(-1)^{|\alpha|}\left\langle g, \eta^{(\alpha)}\right\rangle=\left\langle g^{(\alpha)}, \eta\right\rangle .
\end{aligned}
$$

(iii) $\Rightarrow$ (i). We know that $\sum_{\nu>0} \widetilde{\phi}_{\nu} * \phi_{\nu} * f$ converges in $\mathcal{S}^{\prime}$, thus it remains to be shown that $\sum_{\nu \leq 0} \widetilde{\phi}_{\nu} * \phi_{\nu} * f \in\left(\mathcal{S}_{k}\right)^{\prime}$. Let $\eta \in \mathcal{S}_{k}$. Then $\widehat{\eta}^{(\alpha)}(0)=0$ for $|\alpha| \leq k$ and from Taylor's expansion of $\hat{\eta}$ around 0 we have

$$
\widehat{\eta}(\xi)=\sum_{|\beta|=k+1} \frac{\xi^{\beta}}{\beta !} \int_{0}^{1} \widehat{\eta}^{(\beta)}(t \xi) t^{k} d t=\sum_{|\beta|=k+1} \xi^{\beta} \widehat{g}_{\beta}(\xi),
$$

where $\widehat{g}_{\beta}(\xi)=(1 / \beta !) \int_{0}^{1} \widehat{\eta}^{(\beta)}(t \xi) t^{k} d t$. It follows that

$$
\widehat{\theta}(\xi) \widehat{\eta}(\xi)=\sum_{|\beta|=k+1} \xi^{\beta} \widehat{\theta}(\xi) \widehat{g}_{\beta}(\xi) .
$$

By inverting the Fourier transforms we get $\theta * \eta=\sum_{|\beta|=k+1} \eta_{\beta}^{(\beta)}$, where $\eta_{\beta}:=(-i)^{|\beta|} \theta * g_{\beta} \in \mathcal{S}$. Thus,

$$
\begin{aligned}
\sum_{\nu \leq 0}\left\langle\widetilde{\phi}_{\nu} * \phi_{\nu} * f, \eta\right\rangle & =\sum_{\nu \leq 0}\left\langle\widetilde{\phi}_{\nu} * \phi_{\nu} * f, \theta * \eta\right\rangle \\
& =\sum_{|\beta|=k+1} \sum_{\nu \leq 0}\left\langle\widetilde{\phi}_{\nu} * \phi_{\nu} * f, \eta_{\beta}^{(\beta)}\right\rangle \\
& =\sum_{|\beta|=k+1} \sum_{\nu \leq 0}(-1)^{|\beta|}\left\langle\left(\widetilde{\phi}_{\nu} * \phi_{\nu} * f\right)^{(\beta)}, \eta_{\beta}\right\rangle .
\end{aligned}
$$

Since $\sum_{\nu \leq 0}\left(\widetilde{\phi}_{\nu} * \phi_{\nu} * f\right)^{(\beta)}(|\beta|=k+1)$ converges in $\mathcal{S}^{\prime}$, this shows that $\sum_{\nu \leq 0} \widetilde{\phi}_{\nu} * \phi_{\nu} * f$ defines an element of $\left(\mathcal{S}_{k}\right)^{\prime}$.

Combining Propositions 6.1, 6.2 and Lemma 6.2 we immediately get

Proposition 6.3. If $f \in \mathcal{S}^{\prime}$ satisfies the assumptions of either Proposition 6.1 or 6.2 , then 
(i) There exist polynomials $P_{N}, P \in \mathcal{P}_{k}$ such that

$$
\lim _{N \rightarrow \infty}\left(\sum_{\nu=-N}^{\infty} \widetilde{\phi}_{\nu} * \phi_{\nu} * f+P_{N}\right)=f+P \quad \text { in } \mathcal{S}^{\prime} .
$$

(ii) For every $\alpha \in \mathbb{N}^{d}$ with $|\alpha|>k, \sum_{\nu \leq 0}\left(\widetilde{\phi}_{\nu} * \phi_{\nu} * f\right)^{(\alpha)}$ converges in $\mathcal{S}^{\prime}$.

7. Appendix II: inequalities. Let $f$ be a locally integrable function. The maximal operator $M_{t}, t>0$, is defined by

$$
M_{t}(f)(x):=\left(\sup _{Q \ni x}|Q|^{-1} \int_{Q}|f(y)|^{t} d y\right)^{1 / t},
$$

where the supremum is taken over all cubes with sides parallel to the axes.

It is well known (see [FS]) that if $0<p<\infty, 0<q \leq \infty$, and $0<t<$ $\min \{p, q\}$ then for any sequence $\left(f_{j}\right)_{j \in \mathbb{Z}}$ of functions,

$$
\left\|\left(\sum_{j \in \mathbb{Z}} M_{t}\left(f_{j}\right)^{q}\right)^{1 / q}\right\|_{L_{p}} \leq C\left\|\left(\sum_{j \in \mathbb{Z}}\left|f_{j}\right|^{q}\right)^{1 / q}\right\|_{L_{p}} .
$$

Lemma 7.1. Let $0<t \leq 1$ and $M>d / t$. For any $m \in \mathbb{Z}$, any sequence $\left\{h_{J}\right\}_{J \in D_{m}}$ of complex numbers, and $x \in I \in D$, we have

$$
\begin{aligned}
& \sum_{J \in D_{m}}\left|h_{J}\right|\left(1+\frac{\left|x_{I}-x_{J}\right|}{\max \{\ell(I), \ell(J)\}}\right)^{-M} \\
& \leq C \max \left\{(|I| /|J|)^{1 / t}, 1\right\} M_{t}\left(\sum_{J \in D_{m}}\left|h_{J}\right| \chi_{J}\right)(x) .
\end{aligned}
$$

Proof. Without loss of generality we assume that $x_{I}=0$.

CASE I: $|I| \leq 2^{-m d}$. We let $\delta:=M / d-1 / t>0$, and for each $j \in \mathbb{N}_{+}$ we define $\Omega_{j}:=\left\{J \in D_{m}: 2^{j-1}<2^{m}\left|x_{J}\right| \leq 2^{j}\right\}$, while $\Omega_{0}:=\left\{J \in D_{m}\right.$ : $\left.2^{m}\left|x_{J}\right| \leq 1\right\}$. If $x \in I$ then

$$
\begin{aligned}
& \sum_{J \in D_{m}}\left|h_{J}\right|\left(1+2^{m}\left|x_{J}\right|\right)^{-M}=\sum_{j=0}^{\infty} \sum_{J \in \Omega_{j}}\left|h_{J}\right|\left(1+2^{m}\left|x_{J}\right|\right)^{-M} \\
& \leq C \sum_{j=0}^{\infty} \sum_{J \in \Omega_{j}}\left|h_{J}\right| 2^{-j M}=C \sum_{j=0}^{\infty} 2^{-j d / t-j \delta d} \sum_{J \in \Omega_{j}}\left|h_{J}\right| \\
& \leq C \sup _{j \geq 0} 2^{-j d / t} \sum_{J \in \Omega_{j}}\left|h_{J}\right| \leq C\left(\sup _{j \geq 0} 2^{-j d} \sum_{J \in \Omega_{j}}\left|h_{J}\right|^{t}\right)^{1 / t} \\
& =C\left(\sup _{j \geq 0} 2^{-j d} 2^{m d} \int\left(\sum_{J \in \Omega_{j}}\left|h_{J}\right| \chi_{J}\right)^{t}\right)^{1 / t}
\end{aligned}
$$




$$
\begin{aligned}
& \leq C\left(\sup _{j \geq 0} \frac{1}{\left|\bigcup_{J \in \Omega_{j}} J\right|} \int_{\bigcup_{J \in \Omega_{j}}}\left(\sum_{J \in \Omega_{j}}\left|h_{J}\right| \chi_{J}\right)^{t}\right)^{1 / t} \\
& \leq C M_{t}\left(\sum_{J \in D_{m}}\left|h_{J}\right| \chi_{J}\right)(x) .
\end{aligned}
$$

CASE II: $|I|>2^{-m d}$. Assume that $\ell(I)=2^{-n}, n<m$. For $j \in \mathbb{N}_{+}$we define $\Omega_{j}:=\left\{J \in D_{m}: 2^{j-1}<2^{n}\left|x_{J}\right| \leq 2^{j}\right\}$, while $\Omega_{0}:=\left\{J \in D_{m}\right.$ : $\left.2^{n}\left|x_{J}\right| \leq 1\right\}$. Then for every $x \in I$ we have

$$
\begin{aligned}
& \sum_{J \in D_{m}}\left|h_{J}\right|\left(1+2^{n}\left|x_{J}\right|\right)^{-M}=\sum_{j=0}^{\infty} \sum_{J \in \Omega_{j}}\left|h_{J}\right|\left(1+2^{n}\left|x_{J}\right|\right)^{-M} \\
& \leq C \sum_{j=0}^{\infty} \sum_{J \in \Omega_{j}}\left|h_{J}\right| 2^{-j M}=C \sum_{j=0}^{\infty} 2^{-j d / t-j \delta d} \sum_{J \in \Omega_{j}}\left|h_{J}\right| \\
& \leq C \sup _{j \geq 0} 2^{-j d / t} \sum_{J \in \Omega_{j}}\left|h_{J}\right| \leq C\left(\sup _{j \geq 0} 2^{-j d} \sum_{J \in \Omega_{j}}\left|h_{J}\right|^{t}\right)^{1 / t} \\
& =C\left(\sup _{j \geq 0} 2^{-j d} 2^{m d} \int\left(\sum_{J \in \Omega_{j}}\left|h_{J}\right| \chi_{J}\right)^{t}\right)^{1 / t} \\
& \leq C 2^{(m-n) d / t}\left(\sup _{j \geq 0} \frac{1}{\left|\bigcup_{J \in \Omega_{j}} J\right|} \int_{\bigcup_{J \in \Omega_{j}}}\left(\sum_{J \in \Omega_{j}}\left|h_{J}\right| \chi_{J}\right)^{t}\right)^{1 / t} \\
& \leq C 2^{(m-n) d / t} M_{t}\left(\sum_{J \in D_{m}}\left|h_{J}\right| \chi_{J}\right)(x) .
\end{aligned}
$$

We leave the proof of the following Hardy-type elementary inequality to the reader.

LEMMA 7.2. Let $\theta>0$ and $0<q \leq \infty$. If $a_{n}, b_{n} \geq 0, n \in \mathbb{Z}$, satisfy

$$
0 \leq b_{n} \leq \sum_{m \leq n} 2^{(m-n) \theta} a_{m}
$$

then

$$
\left(\sum_{n \in \mathbb{Z}} b_{n}^{q}\right)^{1 / q} \leq C\left(\sum_{n \in \mathbb{Z}} a_{n}^{q}\right)^{1 / q} .
$$

LEMMA 7.3. Let $m, n \in \mathbb{Z}$ with $m \geq n$. If $J \in D_{n}$ and $M>d$ then

$$
\sum_{I \in D_{m}}\left(1+\frac{\left|x_{I}-x_{J}\right|}{\ell(J)}\right)^{-M} \leq C 2^{(m-n) d} \text {. }
$$


Proof. We have

$$
\sum_{I \in D_{m}}\left(1+\frac{\left|x_{I}-x_{J}\right|}{\ell(J)}\right)^{-M}=2^{(m-n) M} \sum_{j \in \mathbb{Z}^{d}}\left(2^{m-n}+\left|2^{m} x_{J}-j\right|\right)^{-M} .
$$

If we now use the fact that for every $\varrho \geq 1$ and $M>d$,

$$
\sum_{j \in \mathbb{Z}^{d}}(\varrho+|j|)^{-M} \leq C \varrho^{d-M}
$$

the result follows.

Lemma 7.4. Let $M>d, 1 \leq p \leq \infty$ and $m, n \in \mathbb{Z}$ with $m \leq n$. If $\left(d_{J}\right)_{J \in D_{n}}$ is a sequence of complex numbers then

$$
\left(\sum_{I \in D_{m}}\left(\sum_{J \in D_{n}}\left(1+\frac{\left|x_{I}-x_{J}\right|}{\ell(I)}\right)^{-M}\left|d_{J}\right|\right)^{p}\right)^{1 / p} \leq C 2^{(n-m) d / p^{\prime}}\left(\sum_{J \in D_{n}}\left|d_{J}\right|^{p}\right)^{1 / p}
$$

where $1 / p+1 / p^{\prime}=1$.

Proof. We note that for every $I, \Delta \in D_{m}$, and $J \in D_{n}$ with $J \subset \Delta$,

$$
1+\frac{\left|x_{\Delta}-x_{I}\right|}{\ell(I)} \leq C\left(1+\frac{\left|x_{J}-x_{I}\right|}{\ell(I)}\right) \text {. }
$$

Also for every $I \in D_{m}, D_{m}=\left\{\Delta: \Delta=I+j / 2^{-m}, j \in \mathbb{Z}^{d}\right\}$. Using these two facts we find

$$
\begin{aligned}
\left(\sum_{I \in D_{m}}\left(\sum_{J \in D_{n}}\left(1+\frac{\left|x_{I}-x_{J}\right|}{\ell(I)}\right)^{-M}\left|d_{J}\right|\right)^{p}\right)^{1 / p} \\
=\left(\sum_{I \in D_{m}}\left(\sum_{\Delta \in D_{m}} \sum_{\substack{J \in D_{n} \\
J \subset \Delta}}\left(1+\frac{\left|x_{I}-x_{J}\right|}{\ell(I)}\right)^{-M}\left|d_{J}\right|\right)^{p}\right)^{1 / p} \\
\leq C\left(\sum_{I \in D_{m}}\left(\sum_{\Delta \in D_{m}} \sum_{J \in D_{n}}\left(1+\frac{\left|x_{I}-x_{\Delta}\right|}{\ell \subset \Delta}\right)^{-M}\left|d_{J}\right|\right)^{p}\right)^{1 / p} \\
=C\left(\sum_{I \in D_{m}}\left(\sum_{j \in \mathbb{Z}^{d}}(1+|j|)^{-M} \sum_{\substack{J \in D_{n} \\
J \subset I+j / 2^{m}}}\left|d_{J}\right|\right)^{p}\right)^{1 / p} \\
\leq C \sum_{j \in \mathbb{Z}^{d}}(1+|j|)^{-M}\left(\sum_{I \in D_{m}}\left(\sum_{\substack{J \in D_{n} \\
J \subset I+j / 2^{m}}}\left|d_{J}\right|\right)^{p}\right)^{1 / p}
\end{aligned}
$$




$$
\begin{aligned}
& \leq C 2^{(n-m) d / p^{\prime}} \sum_{j \in \mathbb{Z}^{d}}(1+|j|)^{-M}\left(\sum_{I \in D_{m}} \sum_{\substack{J \in D_{n} \\
J \subset I+j / 2^{m}}}\left|d_{J}\right|^{p}\right)^{1 / p} \\
& \leq C 2^{(n-m) d / p^{\prime}}\left(\sum_{J \in D_{n}}\left|d_{J}\right|^{p}\right)^{1 / p},
\end{aligned}
$$

where we used Minkowski's and Hölder's inequalities.

In a similar vein we have (we leave the proof to the reader):

LEMMA 7.5. Let $M>d, 1 \leq p \leq \infty$ and $m, n \in \mathbb{Z}$ with $m \geq n$. If $\left(d_{J}\right)_{J \in D_{n}}$ is a sequence of complex numbers then

$$
\begin{aligned}
\left(\sum_{I \in D_{m}}\left(\sum_{J \in D_{n}}\left(1+\frac{\left|x_{I}-x_{J}\right|}{\ell(J)}\right)^{-M}\left|d_{J}\right|\right)^{p}\right)^{1 / p} & \leq C 2^{(m-n) d / p}\left(\sum_{J \in D_{n}}\left|d_{J}\right|^{p}\right)^{1 / p} .
\end{aligned}
$$

\section{References}

[B] G. Bourdaud, Ondelettes et espaces de Besov, Rev. Mat. Iberoamericana 11 (1995), 477-512.

[CDF] A. Cohen, I. Daubechies and J.-C. Feauveau, Biorthogonal bases of compactly supported wavelets, Comm. Pure Appl. Math. 45 (1992), 485-560.

[Do] D. Donoho, Unconditional bases are optimal bases for data compression and for statistical estimation, Appl. Comput. Harmon. Anal. 1 (1993), 100-115.

[DJKP] D. Donoho, I. Johnstone, G. Kerkyacharian and D. Picard, Wavelet shrinkage: asymptopia? With discussion and a reply by the authors, J. Roy. Statist. Soc. Ser. B 57 (1995), 301-369.

[FS] C. Fefferman and E. Stein, Some maximal inequalities, Amer. J. Math. 93 (1971), 107-115.

[FJ1] M. Frazier and B. Jawerth, Decomposition of Besov spaces, Indiana Univ. Math. J. 34 (1985), 777-799.

[FJ2] - - - A discrete transform and decompositions of distribution spaces, J. Funct. Anal. 93 (1990), 34-170.

[FJW] M. Frazier, B. Jawerth and G. Weiss, Littlewood-Paley Theory and the Study of Function Spaces, CBMS Regional Conf. Ser. in Math. 79, Amer. Math. Soc., 1991.

[HW] E. Hernandez and G. Weiss, A First Course on Wavelets, Stud. Adv. Math., CRC Press, 1996.

[K] G. Kyriazis, Wavelet coefficients measuring smoothness in $H_{p}\left(\mathbb{R}^{d}\right)$, Appl. Comput. Harmon. Anal. 3 (1996), 100-119.

[M] Y. Meyer, Ondelettes et opérateurs I: ondelettes, Hermann, 1990.

[Pee] J. Peetre, New Thoughts on Besov Spaces, Duke Univ. Math. Ser. 1, Math. Dept., Duke Univ., Durham, NC, 1976.

[Pet] P. Petrushev, Bases consisting of rational functions of uniformly bounded degrees or more general functions, J. Funct. Anal. 174 (2000), 18-75. 
[R] W. Rudin, Functional Analysis, McGraw-Hill, 1973.

[T] H. Triebel, Theory of Function Spaces, Birkhäuser, 1983.

Department of Mathematics and Statistics

University of Cyprus

P.O. Box 20537

1678 Nicosia, Cyprus

E-mail: kyriazis@ucy.ac.cy

Received June 10, 2002

Revised version January 21, 2003 